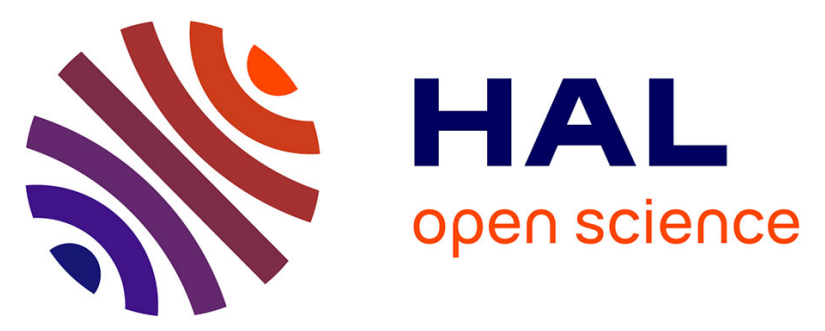

\title{
PERIODIC MOTIONS AND CHAOS WITH IMPACTING CHATTER AND STICK IN A GEAR TRANSMISSION SYSTEM
}

\author{
Albert C. J. Luo, Dennis O'Connor
}

\section{- To cite this version:}

Albert C. J. Luo, Dennis O'Connor. PERIODIC MOTIONS AND CHAOS WITH IMPACTING CHATTER AND STICK IN A GEAR TRANSMISSION SYSTEM. International journal of bifurcation and chaos in applied sciences and engineering , 2009, 19 (6), pp.1975-1994. 10.1142/S0218127409023858 . hal-01509344

\section{HAL Id: hal-01509344 https://hal.science/hal-01509344}

Submitted on 17 Apr 2017

HAL is a multi-disciplinary open access archive for the deposit and dissemination of scientific research documents, whether they are published or not. The documents may come from teaching and research institutions in France or abroad, or from public or private research centers.
L'archive ouverte pluridisciplinaire HAL, est destinée au dépôt et à la diffusion de documents scientifiques de niveau recherche, publiés ou non, émanant des établissements d'enseignement et de recherche français ou étrangers, des laboratoires publics ou privés. 


\title{
PERIODIC MOTIONS AND CHAOS WITH IMPACTING CHATTER AND STICK IN A GEAR TRANSMISSION SYSTEM
}

\author{
ALBERT C. J. LUO* and DENNIS O'CONNOR \\ Department of Mechanical and Industrial Engineering, \\ Southern Illinois University Edwardsville, \\ Edwardsville, IL 62026-1805, USA
}

\begin{abstract}
This paper focuses on periodic motions and chaos relative to the impacting chatter and stick in order to find the origin of noise and vibration in such a gear transmission system. Such periodic motions are predicted analytically through mapping structures, and the corresponding local stability and bifurcation analysis are carried out. The grazing and stick conditions presented in [Luo \& O'Connor, 2007] are adopted to determine the existence of periodic motions, which cannot be achieved from the local stability analysis. Numerical simulations are performed to illustrate periodic motions and stick motion criteria. Such an investigation may provide some clues to reduce the noise in gear transmission systems.
\end{abstract}

Keywords: Discontinuous dynamical systems; gear transmission; impacting chatter; stick motion; grazing bifurcation; periodic motions.

\section{Introduction}

In [Luo \& O'Connor, 2007], the motion mechanism of impacting chatter with stick in the gear transmission dynamical system was discussed. The reduction of noise in such a gear transmission system requires the identification of the existence of periodic motions. Hence, in this paper, periodic motions in such a gear transmission system will be addressed. The early investigation on periodic motions in gear transmission systems can be found in publications in the 1930's. den Hartog and Mikina [1932] gave a solution for symmetric, periodic motion of such a gear system. The used model is a piecewise system without damping. After half a century, Shaw and Holmes [1983] used the mapping technique to investigate periodic motion and stability of a piecewise linear system with a single discontinuity. Natsiavas [1989] investigated the stability and bifurcation of periodic motions in a piecewise linear system with a symmetric trilinear spring. Based on the piecewise linear model, periodic motions for gear transmission systems were extensively investigated in 1990's (e.g. [Comparin \& Singh, 1989; Kahraman \& Singh, 1990; Rook \& Singh, 1995; Theodossiades \& Natsiavas, 2000]). The asymmetric motion of the piecewise linear system with the infinite stiffness was investigated numerically in [Li et al., 1990]. The periodic solutions and bifurcations in the piecewise linear oscillator were further discussed, for which refer to [Klezka et al., 1992]. In recent years, the mapping concepts were extensively adopted to determine the periodic motion in impacting systems (e.g. [Han et al., 1995; Luo, 2002]). This methodology was also employed to investigate the periodic and chaotic motions of the periodically driven piecewise linear systems (e.g. [Luo \& Menon, 2004; Menon \& Luo, 2005]). In [Luo, 2005], a generalized methodology was given 
for predicting arbitrary periodic motions of discontinuous systems. A summary of the generalized method for the analytical prediction of complicated periodic motions in discontinuous systems was presented in [Luo, 2006].

Pfeiffer [1984] presented an impact model of gear transmissions, and the theoretical and experimental investigations on regular and chaotic motions in the gear box were carried out in [Karagiannis \& Pfeiffer, 1991]. Nordmark [1991] investigated the grazing-induced nonperiodic motion of the impacting oscillator, but only the necessary condition for such a grazing motion in the impacting oscillator was given. Furthermore, the normal form mapping for such grazing phenomena was further developed in [di Bernardo et al., 2001, 2002]. To model the gear transmission system, Luo and Chen [2005] proposed a piecewise linear, impacting system to investigate the periodic motion. For such an oscillator, not only the simple periodic motion but also many complicated periodic motions exist. The switching between the two periodic motions causes motion complexity, and the asymmetric periodic motions exhibit the period-doubling bifurcation in such an oscillator. Luo and Chen [2007] used the mapping technique to analytically predict arbitrary periodic motions for such an oscillator. As in $\underline{\underline{L u O}}$ \& O'Connor, 2007], it was stated that the impacting location in the model of Luo and Chen [2007] was fixed and the perfect plastic impact was considered in this piecewise linear model. The separation of two gears occurs at the same location of gear impacts. To improve the modeling of gear transmission systems, the two gears will be considered to be independent, and impacts between the two separated oscillators will be adopted. Based on such a new model, the motion mechanism of the impacting chatter and stick was discussed in $\lceil$ Luo \& O'Connor, 2007]. For the further understanding of the origin of the vibration and noise in gear transmission systems, the corresponding periodic motions for such a new model will be investigated herein. Compared to the earliest results, the characteristics of complicated periodic motions can be predicted analytically by the mapping technique.

In this paper, basic mappings will be introduced first from the separation boundaries, and from the basic mappings, the mapping structures will be developed for any specific periodic motion. Further, the analytical prediction of periodic motions pertaining to impacting chatter and stick will be completed, and the corresponding local stability and bifurcation of the periodic motion will be analyzed. The grazing and stick conditions presented in [Luo \& O'Connor, 2007] will be employed for the existence of periodic motions. Numerical simulations will be carried out for illustration of periodic motions and stick criteria.

\section{Basic Mappings}

For the gear transmission system, equations of motion in the absolute frame are from [Luo \& O'Connor, 2007]

$$
\dot{\mathbf{x}}_{\alpha}^{(i)}=\mathbf{F}_{\alpha}^{(i)}\left(\mathbf{x}_{\alpha}^{(i)}, t\right)
$$

for $i=1,2$ and $\alpha=1,2,3$ with the following vectors

$$
\begin{aligned}
\mathbf{x}_{\alpha}^{(i)} & =\left(x_{\alpha}^{(i)}, \dot{x}_{\alpha}^{(i)}\right)^{T}=\left(x_{\alpha}^{(i)}, y_{\alpha}^{(i)}\right)^{T}, \\
\mathbf{F}_{\alpha}^{(i)} & =\left(\dot{x}_{\alpha}^{(i)}, F_{\alpha}^{(i)}\right)^{T}=\left(y_{\alpha}^{(i)}, F_{\alpha}^{(i)}\right)^{T} ;
\end{aligned}
$$

where

$F_{\alpha}^{(i)}=-2 \zeta_{\alpha}^{(i)} \dot{x}_{\alpha}^{(i)}-\left(\omega_{\alpha}^{(i)}\right)^{2} x_{\alpha}^{(i)}+b_{\alpha}^{(i)}+Q_{\alpha}^{(i)} \cos \Omega t$,

and the superscript " $i$ " represents the $i$ th mass and the subscript " $\alpha$ " represents the $\alpha$-domain. Similarly, the corresponding equations of motion in the relative frame for $i=1,2$ and $\alpha=1,2,3$ are

$$
\dot{\mathbf{z}}_{\alpha}^{(i)}=\mathbf{g}_{\alpha}^{(i)}\left(\mathbf{z}_{\alpha}^{(i)}, \mathbf{x}_{\alpha}^{(\bar{i})}, t\right), \quad \dot{\mathbf{x}}_{\alpha}^{(\bar{i})}=\mathbf{F}_{\alpha}^{(\bar{i})}\left(\mathbf{x}_{\alpha}^{(\bar{i})}, t\right)
$$

where $i, \bar{i} \in\{1,2\}$ and $i \neq \bar{i}$, and the relative vectors are

$$
\left.\begin{array}{l}
\mathbf{z}_{\alpha}^{(i)}=\left(z_{\alpha}^{(i)}, \dot{z}_{\alpha}^{(i)}\right)^{T}=\left(z_{\alpha}^{(i)}, v_{\alpha}^{(i)}\right)^{T}, \\
\mathbf{g}_{\alpha}^{(i)}=\left(\dot{z}_{\alpha}^{(i)}, g_{\alpha}^{(i)}\right)^{T}=\left(v_{\alpha}^{(i)}, g_{\alpha}^{(i)}\right)^{T}
\end{array}\right\}
$$

where

$$
\begin{aligned}
g_{\alpha}^{(i)}= & -2 \zeta_{\alpha}^{(i)} \dot{z}_{\alpha}^{(i)}-\left(\omega_{\alpha}^{(i)}\right)^{2} z_{\alpha}^{(i)}+b_{\alpha}^{(i)}+Q_{\alpha}^{(i)} \cos \Omega t \\
& -\ddot{x}_{\alpha}^{(\bar{i})}-2 \zeta_{\alpha}^{(i)} \dot{x}_{\alpha}^{(\bar{i})}-\left(\omega_{\alpha}^{(i)}\right)^{2} x_{\alpha}^{(\bar{i})} .
\end{aligned}
$$

To investigate periodic impacting chatter with/ without stick in the gear transmission system, the mapping structure will be developed from separation boundaries in [Luo \& O'Connor, 2007]. Before the mapping structure for a prescribed impacting chatter motion is developed, the switching planes will be defined first, and from the switching planes, the basic mappings can be developed for the gear transmission system.

From discontinuous boundaries in $[$ Luo \& O'Connor, 2007], the switching planes based on the 
two impacting chatter boundaries are defined as

$$
\begin{aligned}
& R_{\Sigma_{2 \infty}^{(i)}}=\left\{\begin{array}{l|l}
\left(t_{k}, x_{k}^{(i)}, \dot{x}_{k}^{(i)}, \dot{x}_{k}^{(\bar{i})}\right) & \begin{array}{l}
x_{k}^{(\bar{i})}=x_{k}^{(i)}-\frac{d}{2} \\
\dot{x}_{k}^{(i)} \neq \dot{x}_{k}^{(\bar{i})}
\end{array}
\end{array}\right\}, \\
& { }^{L} \Sigma_{2 \infty}^{(i)}=\left\{\begin{array}{l|l}
\left(t_{k}, x_{k}^{(i)}, \dot{x}_{k}^{(i)}, \dot{x}_{k}^{(\bar{i})}\right) & \begin{array}{l}
x_{k}^{(\bar{i})}=x_{k}^{(i)}+\frac{d}{2} \\
\dot{x}_{k}^{(i)} \neq \dot{x}_{k}^{(\bar{i})}
\end{array}
\end{array}\right\} \cdot
\end{aligned}
$$

From now on, $x_{k}^{(i)} \equiv x^{(i)}\left(t_{k}\right)$ and $\dot{x}_{k}^{(i)} \equiv \dot{x}^{(i)}\left(t_{k}\right)$ on the separation boundary at time $t_{k}$ are switching displacement and velocity in the absolute frame. The switching phase is defined by $\varphi_{k}=$ $\bmod \left(\Omega t_{k}, 2 \pi\right)$. In the relative frame, the switching planes are expressed as

$$
\left.\begin{array}{l}
R_{\Sigma_{2 \infty}^{(i)}}=\left\{\left(t_{k}, \dot{z}_{k}^{(i)}, x_{k}^{(\bar{i})}, \dot{x}_{k}^{(\bar{i})}\right) \mid z_{k}^{(i)}=\frac{d}{2}, \dot{z}_{k}^{(i)} \neq 0\right\} \\
{ }^{L} \Sigma_{2 \infty}^{(i)}=\left\{\left(t_{k}, \dot{z}_{k}^{(i)}, x_{k}^{(\bar{i})}, \dot{x}_{k}^{(\bar{i})}\right) \mid z_{k}^{(i)}=-\frac{d}{2}, \dot{z}_{k}^{(i)} \neq 0\right\}
\end{array}\right\}
$$

The two switching sets are then decomposed as

$$
\begin{gathered}
R_{\Sigma_{2 \infty}^{(i)}}^{(i)}{ }_{+}^{R} \Sigma_{2 \infty}^{(i)} \cup{ }_{-}^{R} \Sigma_{2 \infty}^{(i)} \text { and } \\
{ }_{\Sigma_{2 \infty}}^{(i)}={ }_{+}^{L} \Sigma_{2 \infty}^{(i)} \cup{ }_{-}^{L} \Sigma_{2 \infty}^{(i)}
\end{gathered}
$$

where

$$
\begin{aligned}
& { }_{+}^{R} \Sigma_{2 \infty}^{(i)}=\left\{\left(t_{k}, \dot{z}_{k}^{(i)}, x_{k}^{(\bar{i})}, \dot{x}_{k}^{(\bar{i})}\right) \mid z_{k}^{(i)}=\frac{d}{2}, \dot{z}_{k}^{(i)}>0\right\}, \\
& \underline{-}_{\Sigma_{2 \infty}^{(i)}}=\left\{\left(t_{k}, \dot{z}_{k}^{(i)}, x_{k}^{(\bar{i})}, \dot{x}_{k}^{(\bar{i})}\right) \mid z_{k}^{(i)}=\frac{d}{2}, \dot{z}_{k}^{(i)}<0\right\} ; \\
& { }_{+}^{R} \Sigma_{2 \infty}^{(i)}=\left\{\left(t_{k}, \dot{z}_{k}^{(i)}, x_{k}^{(\bar{i})}, \dot{x}_{k}^{(\bar{i})}\right) \mid z_{k}^{(i)}=-\frac{d}{2}, \dot{z}_{k}^{(i)}>0\right\}, \\
& { }_{-}^{R} \Sigma_{2 \infty}^{(i)}=\left\{\left(t_{k}, z_{k}^{(i)}, x_{k}^{(\bar{i})}, \dot{x}_{k}^{(\bar{i})}\right) \mid z_{k}^{(i)}=-\frac{d}{2}, \dot{z}_{k}^{(i)}<0\right\} .
\end{aligned}
$$

Based on the above definitions of switching planes, four mappings are defined in the absolute frame as

$$
\left.\begin{array}{c}
P_{2}:{ }^{R} \Sigma_{2 \infty}^{(i)} \rightarrow{ }^{R} \Sigma_{2 \infty}^{(i)}, \quad P_{3}:{ }^{R} \Sigma_{2 \infty}^{(i)} \rightarrow{ }^{L} \Sigma_{2 \infty}^{(i)} ; \\
P_{5}:{ }^{L} \Sigma_{2 \infty}^{(i)} \rightarrow{ }^{L} \Sigma_{2 \infty}^{(i)}, \quad P_{6}:{ }^{L} \Sigma_{2 \infty}^{(i)} \rightarrow{ }^{R} \Sigma_{2 \infty}^{(i)}
\end{array}\right\}
$$

and in the relative frame, the mappings are also defined as

$$
\left.\begin{array}{l}
P_{2}:{ }_{-}^{R} \Sigma_{2 \infty}^{(i)} \rightarrow{ }_{+}^{R} \Sigma_{2 \infty}^{(i)}, \quad P_{3}:{ }_{-}^{R} \Sigma_{2 \infty}^{(i)} \rightarrow{ }_{-}^{L} \Sigma_{2 \infty}^{(i)} ; \\
P_{5}:{ }_{+}^{L} \Sigma_{2 \infty}^{(i)} \rightarrow{ }_{-}^{L} \Sigma_{2 \infty}^{(i)}, \quad P_{6}:{ }_{+}^{L} \Sigma_{2 \infty}^{(i)} \rightarrow{ }_{+}^{R} \Sigma_{2 \infty}^{(i)} .
\end{array}\right\}
$$

Among four basic mappings, the two mappings $\left(P_{2}\right.$ and $\left.P_{4}\right)$ are local and the other two mappings $\left(P_{3}\right.$ and $\left.P_{6}\right)$ are global. The local mapping will map the motion from a switching plane onto itself. However,

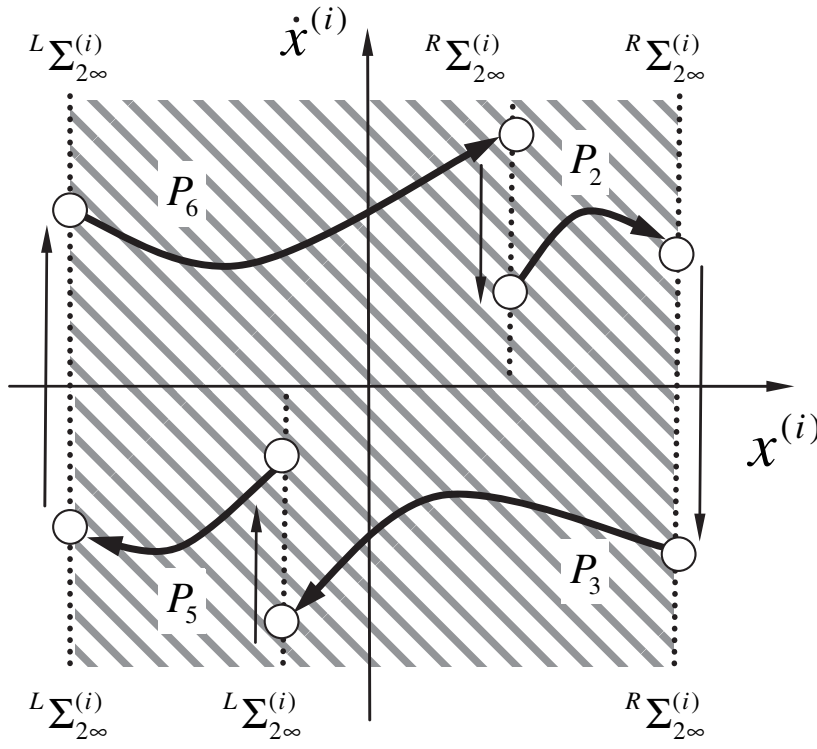

(a)

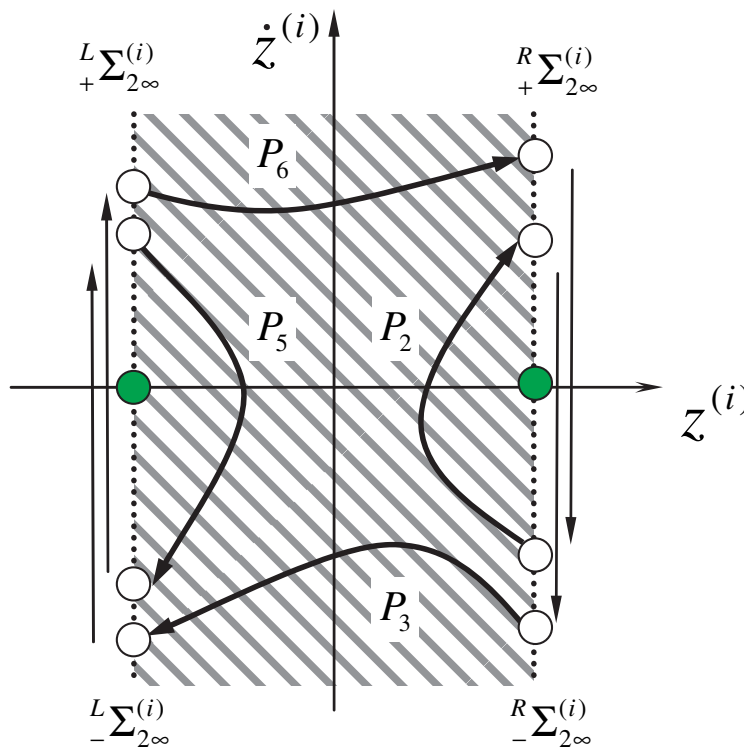

(b)

Fig. 1. Basic mappings for impacting chatter in (a) the absolute frame and (b) the relative frame. The straight line with arrow represents an impact on the boundary. 
the global mapping will map the motion from a switching plane to another one. Such mappings are sketched in Fig. 1. The corresponding switching planes are labeled. The mappings for the absolute and relative frames are arranged in Figs. 1(a) and 1(b), respectively. On the impacting chatter boundaries, impacts are expressed by thin straight lines with arrows.

To investigate stick motions in the gear transmission system, the switching planes for stick are defined as

$$
\begin{aligned}
& \Sigma_{12}^{(i)}=\left\{\left(t_{k}, x_{k}^{(i)}, \dot{x}_{k}^{(i)}, \dot{x}_{k}^{(\bar{i})}\right) \mid x_{k}^{(i)}={ }^{R} x_{2-}^{(\bar{i})}+\frac{d}{2}, \dot{x}_{k}^{(i)}={ }^{R} \dot{x}_{2-}^{(\bar{i})}\right\}, \\
& \Sigma_{21}^{(i)}=\left\{\left(t_{k}, x_{k}^{(i)}, \dot{x}_{k}^{(i)}, \dot{x}_{k}^{(\bar{i})}\right) \mid x_{k}^{(i)}={ }^{R} x_{2+}^{(\bar{i})}+\frac{d}{2}, \dot{x}_{k}^{(i)}={ }^{R} \dot{x}_{2+}^{(\bar{i})}\right\} ; \\
& \Sigma_{23}^{(i)}=\left\{\left(t_{k}, x_{k}^{(i)}, \dot{x}_{k}^{(i)}, \dot{x}_{k}^{(\bar{i})}\right) \mid x_{k}^{(i)}={ }^{L} x_{2-}^{(\bar{i})}+\frac{d}{2}, \dot{x}_{k}^{(i)}={ }^{L} \dot{x}_{2-}^{(\bar{i})}\right\}, \\
& \Sigma_{32}^{(i)}=\left\{\left(t_{k}, x_{k}^{(i)}, \dot{x}_{k}^{(i)}, \dot{x}_{k}^{(\bar{i})}\right) \mid x_{k}^{(i)}={ }^{L} x_{2+}^{(\bar{i})}+\frac{d}{2}, \dot{x}_{k}^{(i)}={ }^{L} \dot{x}_{2+}^{(\bar{i})}\right\}
\end{aligned}
$$

for the absolute frame and

$$
\left.\begin{array}{l}
\Sigma_{12}^{(i)}=\left\{\left(t_{k}, \dot{z}_{k}^{(i)}, x_{k}^{(\bar{i})}, \dot{x}_{k}^{(\bar{i})}\right) \mid z_{k}^{(i)}=\frac{d}{2}, \dot{z}_{k}^{(i)}=0_{-}\right\}, \\
\Sigma_{21}^{(i)}=\left\{\left(t_{k}, \dot{z}_{k}^{(i)}, x_{k}^{(\bar{i})}, \dot{x}_{k}^{(\bar{i})}\right) \mid z_{k}^{(i)}=\frac{d}{2}, \dot{z}_{k}^{(i)}=0_{+}\right\} ; \\
\Sigma_{23}^{(i)}=\left\{\left(t_{k}, \dot{z}_{k}^{(i)}, x_{k}^{(\bar{i})}, \dot{x}_{k}^{(\bar{i})}\right) \mid z_{k}^{(i)}=-\frac{d}{2}, \dot{z}_{k}^{(i)}=0_{-}\right\}, \\
\Sigma_{32}^{(i)}=\left\{\left(t_{k}, \dot{z}_{k}^{(i)}, x_{k}^{(\bar{i})}, \dot{x}_{k}^{(\bar{i})}\right) \mid z_{k}^{(i)}=-\frac{d}{2}, \dot{z}_{k}^{(i)}=0_{+}\right\}
\end{array}\right\}
$$

for the relative frame.

In the relative frame, the switching planes in Eqs. (10) and (13) are almost same. In Eq. (10), the relative velocity is nonzero (i.e. $\dot{z}_{k}^{(i)} \neq 0$ ) but in Eq. (13), the relative velocity is zero (i.e. $\dot{z}_{k}^{(i)}=0$ ). The two switching planes can be treated as the same for all mappings. Except for two stick mappings (i.e. $P_{1}$ and $P_{4}$ ), the other mappings are the same as in Eqs. (11) and (12). From the stick switching planes, the mappings are defined as

$$
\left.\begin{array}{l}
P_{1}: \Sigma_{21}^{(i)} \rightarrow \Sigma_{12}^{(i)}, \quad P_{2}: \Sigma_{12}^{(i)} \rightarrow \Sigma_{21}^{(i)}, \quad P_{3}: \Sigma_{12}^{(i)} \rightarrow \Sigma_{23}^{(i)} ; \\
P_{4}: \Sigma_{23}^{(i)} \rightarrow \Sigma_{32}^{(i)}, \quad P_{5}: \Sigma_{23}^{(i)} \rightarrow \Sigma_{32}^{(i)}, \quad P_{6}: \Sigma_{32}^{(i)} \rightarrow \Sigma_{21}^{(i)}
\end{array}\right\}
$$

With mixed switching planes, six mappings are defined by

$$
\begin{aligned}
& P_{2}: \Sigma_{12}^{(i)} \rightarrow{ }^{R} \Sigma_{2 \infty}^{(i)}, \quad P_{2}:{ }^{R} \Sigma_{2 \infty}^{(i)} \rightarrow \Sigma_{21}^{(i)} ; \\
& P_{3}: \Sigma_{12}^{(i)} \rightarrow{ }^{L} \Sigma_{2 \infty}^{(i)}, \quad P_{3}:{ }^{R} \Sigma_{2 \infty}^{(i)} \rightarrow \Sigma_{23}^{(i)} \\
& P_{5}: \Sigma_{23}^{(i)} \rightarrow{ }^{L} \Sigma_{2 \infty}^{(i)}, \quad P_{5}:{ }^{L} \Sigma_{2 \infty}^{(i)} \rightarrow \Sigma_{32}^{(i)} \text {, } \\
& P_{6}: \Sigma_{32}^{(i)} \rightarrow{ }^{R} \Sigma_{2 \infty}^{(i)}, \quad P_{6}:{ }^{L} \Sigma_{2 \infty}^{(i)} \rightarrow \Sigma_{21}^{(i)} .
\end{aligned}
$$

Similarly, the mappings based on the relative switching planes can be defined. The mappings relative to the stick switching planes only are sketched in Fig. 2. In Fig. 2(a), only two stick mappings $\left(P_{1}\right.$ and $\left.P_{4}\right)$ are new, and the other four mappings are the same as in Fig. 1 . In the relative frame, the switching planes are points. The stick mapping is very difficult to be illustrated. The other mappings are presented in Figs. 2(b). The mappings based on the sticking and impacting switching planes are presented in Figs. 3(a) and 3(b).

Set the vectors as

$$
\left.\begin{array}{l}
\mathbf{y}_{k} \equiv\left(t_{k}, x_{k}^{(i)}, \dot{x}_{k}^{(i)}, \dot{x}_{k}^{(\bar{i})}\right)^{T}, \\
\mathbf{w}_{k} \equiv\left(t_{k}, \dot{z}_{k}^{(i)}, x_{k}^{(\bar{i})}, \dot{x}_{k}^{(\bar{i})}\right)^{T} .
\end{array}\right\}
$$




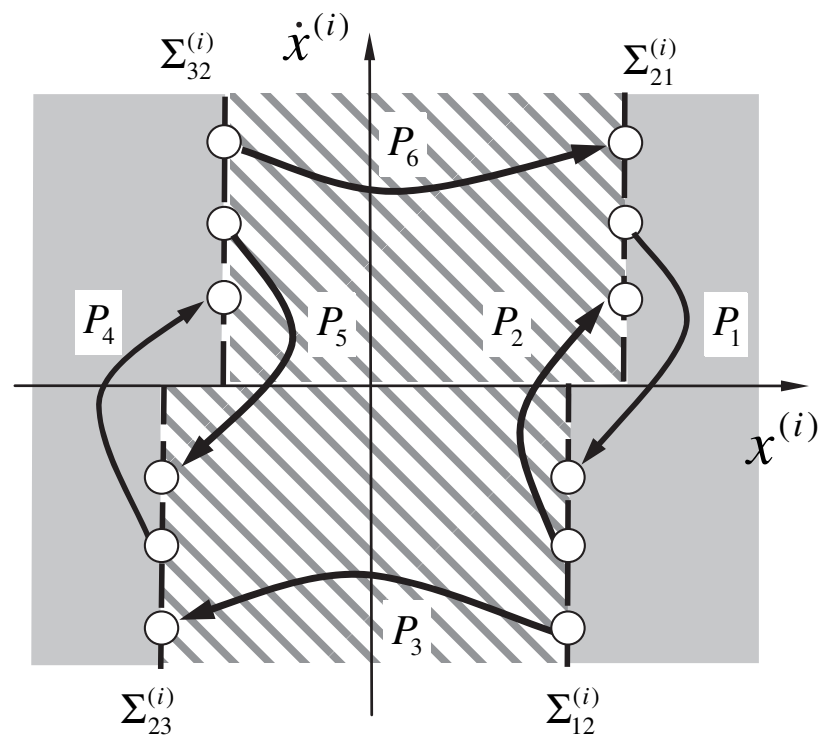

(a)

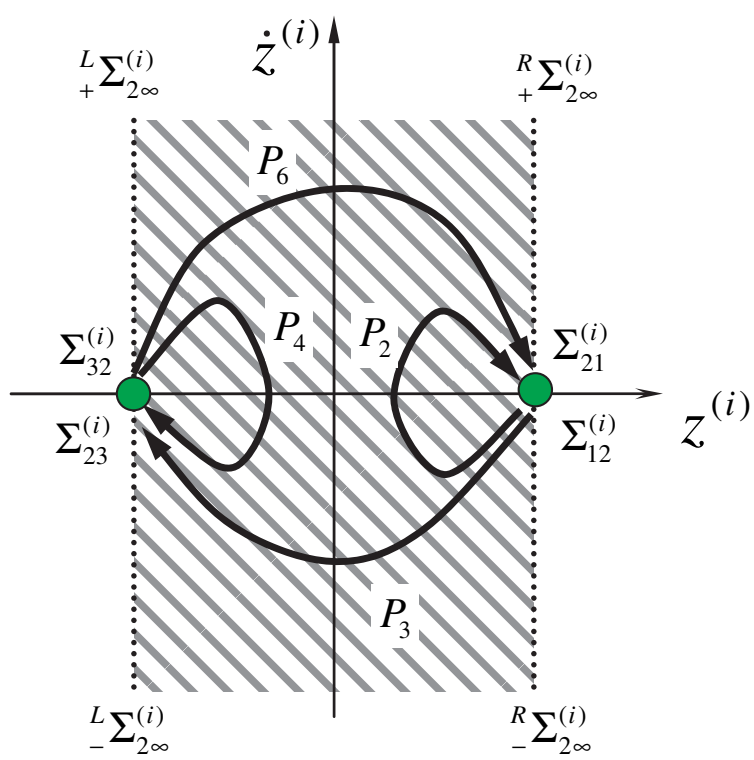

(b)

Fig. 2. Mappings from stick switching planes: (a) absolute motion and (b) relative motion.

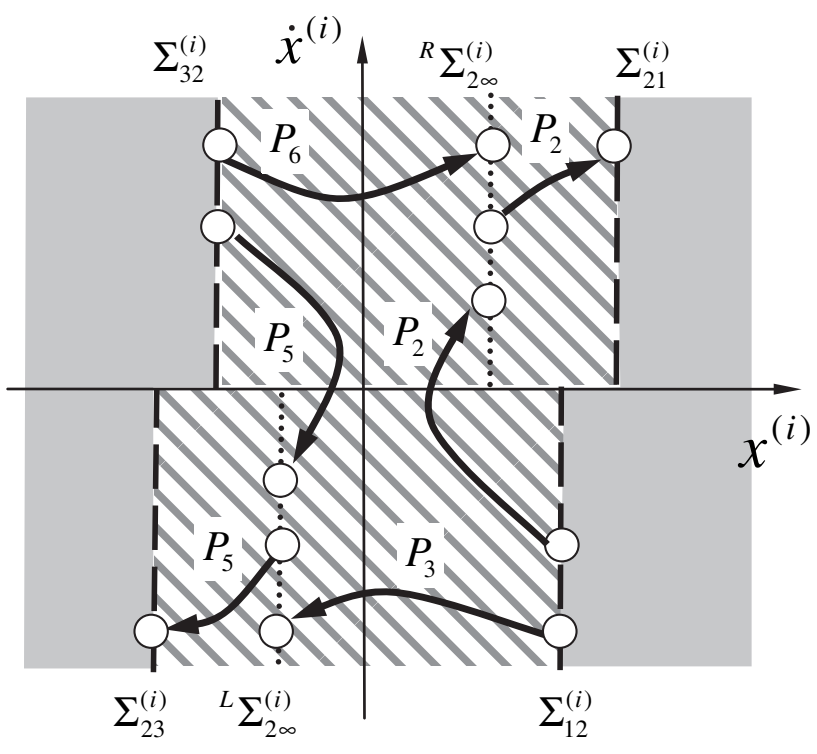

(a)

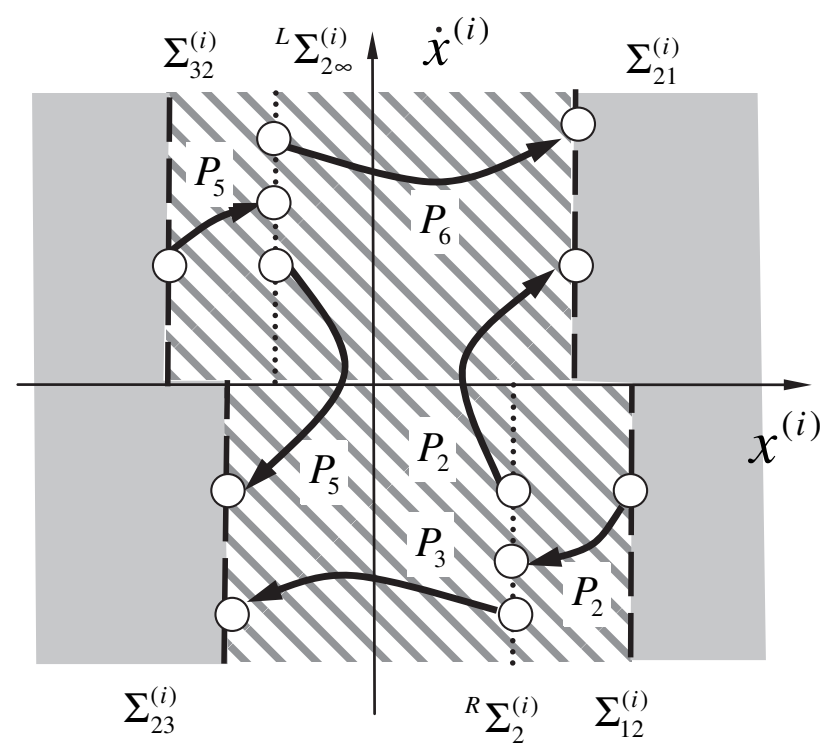

(b)

Fig. 3. Mappings between switching planes for stick and impacting.

For the impacting maps $P_{\sigma}(\sigma=2,3,5,6)$ in the absolute coordinate, $\mathbf{y}_{k+1}=P_{\sigma} \mathbf{y}_{k}$ can be expressed by

$$
P_{\sigma}:\left(t_{k}, x_{k}^{(i)}, \dot{x}_{k}^{(i)}, \dot{x}_{k}^{(\bar{i})}\right) \rightarrow\left(t_{k+1}, x_{k+1}^{(i)}, \dot{x}_{k+1}^{(i)}, \dot{x}_{k+1}^{(\bar{i})}\right)
$$

From Appendix, the absolute displacement and velocity for two gear oscillators can be obtained with initial conditions $\left(t_{k}, x_{k}^{(i)}, \dot{x}_{k}^{(i)}\right)$ and $\left(t_{k}, x_{k}^{(\bar{i})}, \dot{x}_{k}^{(\bar{i})}\right)$. The final state for time $t_{k+1}$ can be given. The switching planes give $x_{\gamma}^{(\bar{i})}=x_{\gamma}^{(i)} \pm(d / 2)$ $(\gamma=k, k+1)$. The four equations of displacement and velocity for two gears give a set of four algebraic equations, i.e.

$$
\mathbf{f}^{(\sigma)}\left(\mathbf{y}_{k}, \mathbf{y}_{k+1}\right)=0
$$

where

$$
\mathbf{f}^{(\sigma)}=\left(f_{1}^{(\sigma)}, f_{2}^{(\sigma)}, f_{3}^{(\sigma)}, f_{4}^{(\sigma)}\right)^{T}
$$


For the stick motion, the displacement and velocity of the $i$ th-gear will be adopted. In addition, the following equations are used.

$\dot{x}_{k+1}^{(\bar{i})}=\dot{x}_{k+1}^{(i)}, g_{\alpha}^{(i)}\left(x_{k+1}^{(\bar{i})}, \dot{x}_{k+1}^{(\bar{i})}, \ddot{x}_{k+1}^{(\bar{i})}, t_{k+1}\right)=0$.

With the condition $x_{\gamma}^{(\bar{i})}=x_{\gamma}^{(i)} \pm(d / 2)(\gamma=k, k+1)$, the algebraic equations in Eq. (19) can be obtained. If a mapping only starts or ends at the stick boundary, the corresponding displacement plus the following equation can be employed to obtain Eq. (19).

$$
\dot{x}_{\gamma}^{(\bar{i})}=\dot{x}_{\gamma}^{(i)}, \quad \text { for } \gamma \in\{k, k+1\} .
$$

Based on the relative coordinate $\left(z^{(i)}, \dot{z}^{(i)}\right)$, the relative displacement and velocity can be used with the initial condition $\left(t_{k}, z_{k}^{(i)}, \dot{z}_{k}^{(i)}\right)$ on the switching boundary. The displacement and velocity with an initial condition $\left(t_{k}, x_{k}^{(\bar{i})}, \dot{x}_{k}^{(\bar{i})}\right)$ can be given. With conditions $z_{\gamma}^{(i)}= \pm(d / 2)(\gamma=k, k+1)$, the relative and absolute displacements and velocities generate a set of four algebraic equations as

$$
\mathbf{h}^{(\sigma)}\left(\mathbf{w}_{k}, \mathbf{w}_{k+1}\right)=0
$$

where

$$
\mathbf{h}^{(\sigma)}=\left(h_{1}^{(\sigma)}, h_{2}^{(\sigma)}, h_{3}^{(\sigma)}, h_{4}^{(\sigma)}\right)^{T} .
$$

In a similar fashion, for stick mapping, we have

$$
\left.\begin{array}{l}
\dot{z}_{k+1}^{(i)}=\dot{z}_{k}^{(i)}=0, \\
g_{\alpha}^{(i)}\left(x_{k+1}^{(\bar{i})}, \dot{x}_{k+1}^{(\bar{i})}, \ddot{x}_{k+1}^{(\bar{i})}, t_{k+1}\right)=0 .
\end{array}\right\}
$$

If a mapping only starts or ends at the stick boundary, the relative displacement plus the following equation can be used to obtain Eq. (23).

$$
\dot{z}_{\gamma}^{(i)}=0, \quad \text { for } \gamma \in\{k, k+1\} .
$$

Finally, from Luo and O'Connor (1), the impact mapping on the impact boundaries is defined as

$$
P_{0}:{ }^{R} \Sigma_{2 \infty}^{(i)} \rightarrow{ }^{R} \Sigma_{2 \infty}^{(i)} \quad \text { and } \quad P_{0}:{ }^{L} \Sigma_{2 \infty}^{(i)} \rightarrow{ }^{L} \Sigma_{2 \infty}^{(i)}
$$

in the absolute frame and

$$
P_{0}:{ }_{+}^{R} \Sigma_{2 \infty}^{(i)} \rightarrow{ }_{-}^{R} \Sigma_{2 \infty}^{(i)} \text { and } P_{0}:{ }_{-}^{L} \Sigma_{2 \infty}^{(i)} \rightarrow{ }_{+}^{L} \Sigma_{2 \infty}^{(i)}
$$

in the relative frame. The corresponding functions in Eqs. (19) and (23), respectively, are

$$
\left.\begin{array}{l}
f_{1}^{(0)}=t_{k+1}-t_{k}, \\
f_{2}^{(0)}=x_{k+1}^{(i)}-x_{k}^{(i)}, \\
f_{3}^{(0)}=\dot{x}_{k+1}^{(i)}-I_{1}^{(i)} \dot{x}_{k}^{(i)}-I_{2}^{(i)} \dot{x}_{k}^{(\bar{i})}, \\
f_{4}^{(0)}=\dot{x}_{k+1}^{(\bar{i})}-I_{1}^{(\bar{i})} \dot{x}_{k}^{(\bar{i})}-I_{2}^{(\bar{i})} \dot{x}_{k}^{(i)} \cdot
\end{array}\right\}
$$

$h_{1}^{(0)}=t_{k+1}-t_{k}$,

$h_{2}^{(0)}=\dot{z}_{k+1}^{(i)}+e \dot{z}_{k}^{(i)}$,

$h_{3}^{(0)}=x_{k+1}^{(\bar{i})}-x_{k}^{(\bar{i})}$,

$h_{4}^{(0)}=\dot{x}_{k+1}^{(\bar{i})}-\dot{x}_{k}^{(\bar{i})}-\frac{m_{i}}{m_{1}+m_{2}}\left(\dot{z}_{k+1}^{(i)}-\dot{z}_{k}^{(i)}\right)$.

For simplicity of mapping structures of periodic motions, the impact mapping will be dropped from now on, but the impact relation will be embedded.

\section{Mapping Structures}

To investigate periodic motions in such a gear transmission system, the notation for mapping actions of basic mappings is introduced as in [Luo, 2005, 2006]

$$
P_{n_{k} \cdots n_{2} n_{1}} \equiv P_{n_{k}} \circ \cdots \circ P_{n_{2}} \circ P_{n_{1}}
$$

where the mapping $P_{n_{j}}\left(n_{j} \in\{1,2, \ldots, 6\}, j=\right.$ $1,2, \ldots, k)$ is defined in the previous section. Consider a generalized mapping structure as

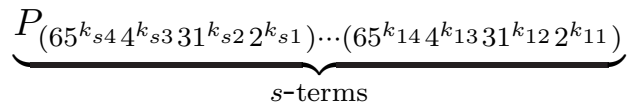

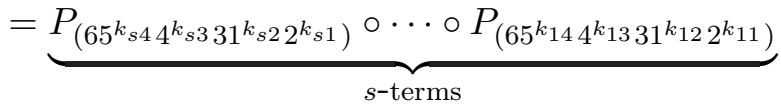

where $\left(k_{\mu \nu} \in\{0, \mathbb{N}\}, \mu=1,2, \ldots, s, \nu=1,2,3,4\right)$. From the generalized mapping structure, consider a simple mapping structure of periodic motions for impacting chatter. For instance, the mapping structure is

$$
P_{65^{n} 32^{m}}=P_{6} \circ P_{5^{n}} \circ P_{3} \circ P_{2^{m}}
$$

where $m, n \in\{0, \mathbb{N}\}$. Such a mapping structure gives $(m+1)$-impacts in boundary ${ }^{R} \partial \Omega_{2 \infty}^{(i)}$ and $(n+1)$-impacts in boundary ${ }^{L} \partial \Omega_{2 \infty}^{(i)}$, which are described by mappings $P_{2}$ and $P_{5}$, respectively. Through the global mappings $P_{3}$ and $P_{6}$, the impacting chatters on the two boundaries are connected together. Consider a periodic motion of $P_{65^{n}} 32^{m}$ with period $T_{1}=k_{1} T\left(k_{1} \in \mathbb{N}\right)$. If the mapping structure copies itself, the new mapping structure is:

$$
P_{\left(65^{n} 32^{m}\right)^{2}}=P_{65^{n} 32^{m}} \circ P_{65^{n} 32^{m}}
$$

The periodic motion of $P_{\left(65^{n} 32^{m}\right)^{2}}$ is obtained during a period of $2 T_{1}$. In an alike fashion, such an 
action of mapping structure continues to copy itself with period- $2^{l} T_{1}$.

$$
P_{\left(65^{n} 32^{m}\right)^{2^{l}}}=P_{\left(65^{n} 32^{m}\right)^{2^{l-1}}} \circ P_{\left(65^{n} 32^{m}\right)^{2^{l-1}}}
$$

As $l \rightarrow \infty$, a chaotic motion relative to mapping structure $P_{65^{n}} 32^{m}$ is formed. The prescribed chaos is generated by period-doubling. However, if the grazing bifurcation occurs, such a mapping structure may not be copied by itself. The new mapping structures are combined by the two different mapping structures. For instance,

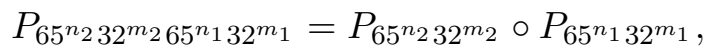

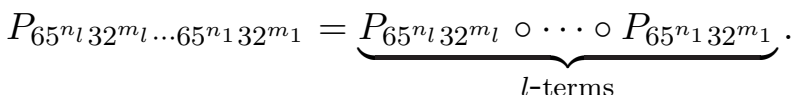

Such a gazing bifurcation will cause the discontinuity of periodic motions, and chaotic motions may exist between periodic motions of $P_{65^{n} l} 32^{m_{l} \ldots 65^{n_{1}}} 32^{m_{1}}$ and $P_{65^{n-1}} 32^{m_{l-1} \ldots 65^{n_{1}}} 32^{m_{1}}$.

For low excitation frequency, the impacting chatter accompanying stick motion exists in the gear transmission system. Consider a simple chatter with stick motion with the following mapping structure

$$
P_{645^{n} 312^{m}}=P_{6} \circ P_{4} \circ P_{5^{n}} \circ P_{3} \circ P_{1} \circ P_{2^{m}}
$$

From the above mapping structure, $m$-impacts in the boundary ${ }^{R} \partial \Omega_{2 \infty}^{(i)}$ and $n$-impacts in the boundary ${ }^{L} \partial \Omega_{2 \infty}^{(i)}$, are described by mappings $P_{2}$ and $P_{5}$, respectively. In addition, both the $m$ th mapping of $P_{2}$ and the $n$th mapping of $P_{5}$ map the impacting boundary to the stick boundary, and the corresponding stick mappings are $P_{1}$ and $P_{4}$, respectively. The two global mappings $P_{3}$ and $P_{6}$ connect the impact and stick boundaries. Similarly, for period-doubling, the mapping structures are given by

$$
\begin{aligned}
& P_{\left(645^{n} 312^{m}\right)^{2}}=P_{645^{n} 312^{m}} \circ P_{645^{n} 312^{m}},
\end{aligned}
$$

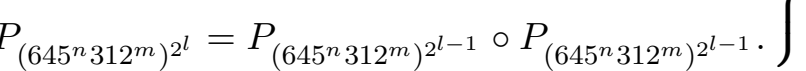

Due to grazing bifurcation, the mapping structures are:

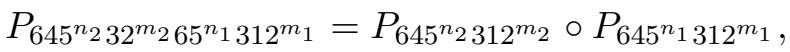

$$
\begin{aligned}
& P_{645^{n} l} 312^{m_{l} \ldots 645^{n_{1}} 312^{m_{1}}}
\end{aligned}
$$

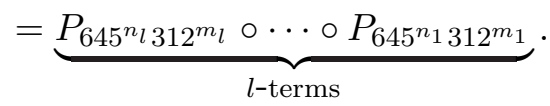

To help one understand two sorts of mapping structures, the two simple mapping structures are shown in Figs. 4(a) and 4(b) for the impacting chatter with and without stick of two gear systems. Similarly, the other mapping structures can be discussed through the generalized mapping structure in Eq. (32). Further, the periodic and chaotic motions relative to a certain mapping structure can be determined.

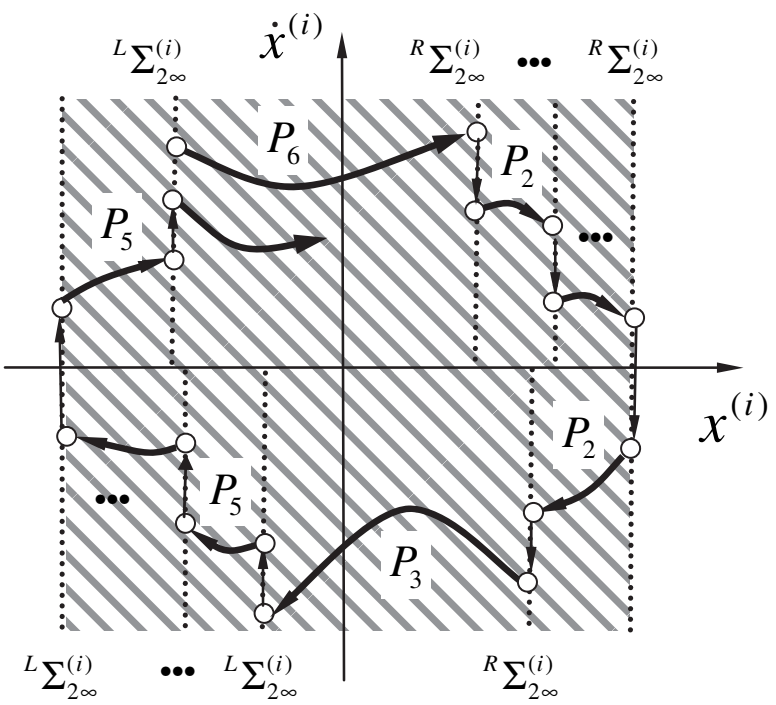

(a)

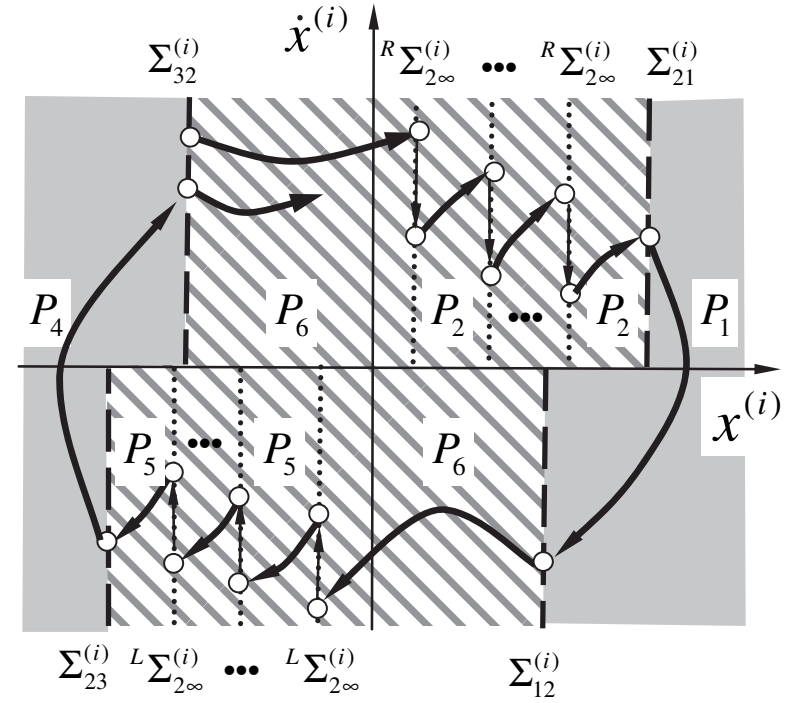

(b)

Fig. 4. Mapping structures for (a) impacting chatter and (b) impacting chatter with stick motion of two gear systems. 


\section{Bifurcation Scenario}

Before discussing impacting chatter in the gear transmission system, a bifurcation scenario is presented through the switching displacements, velocities and phases of two gear oscillators. All the numerical computations are completed from the closed-form solutions in Appendix. The parameters $\left(m_{1}=2, m_{2}=1, r_{1}=r_{2}=0.6, k_{1}=\right.$ $30, k_{2}=20, Q_{0}=30, d=1.0$ and $e=0.7$ ) are considered. The impacting chatter with and without stick varying with excitation frequency are presented in Figs. 5(a)-5(d). The "C-chatter" and "S-chatter" represent the complicated and simple impacting chatters, respectively. The "Chatter w/stick" denotes the impacting chatter with stick for periodic motions. For high excitation frequency, it is observed that the impacting chatter motion possesses simple mapping structure, and the motion is very simple. The excitation frequency lies in the interval of $\Omega \in(4.159,16.433)$ for the simplest, impacting-chatter, periodic motion relative to $P_{63}$. However, the complex, impacting-chatter motion exists for $\Omega \in(1.398,4.159)$. For $\Omega \in(0,1.398)$, the impacting chatter with stick exists. The details of mapping structures for impacting chatter with stick are tabulated in Table 1 , and the excitation frequency range for impacting chatter is in Table 2.

\subsection{Periodic motion and stability}

From mapping structures of periodic motions, the switching sets for any periodic motion can be determined through solving a set of nonlinear algebraic equations. Consider a periodic motion of mapping

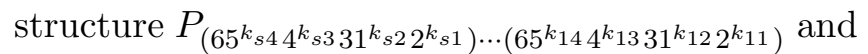
the following relation holds.

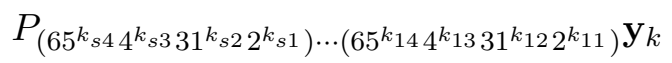

$$
\begin{aligned}
& =\mathbf{y}_{k+2 s+\sum_{m=1}^{s} \sum_{j=1}^{4} k_{m j}}
\end{aligned}
$$

where $\mathbf{y}_{k}=\left(t_{k}, x_{k}^{(i)}, \dot{x}_{k}^{(i)}, \dot{x}_{k}^{(\bar{i})}\right)^{T}$. A set of vector equations is as

$$
\left.\begin{array}{l}
\mathbf{f}^{(2)}\left(\mathbf{y}_{k+1}, \mathbf{y}_{k}\right)=0, \\
\mathbf{f}^{(2)}\left(\mathbf{y}_{k+2}, \mathbf{y}_{k+1}\right)=0, \\
\vdots \\
\mathbf{f}^{(6)}\left(\mathbf{y}_{k+2 s+\sum_{m=1}^{s} \sum_{j=1}^{4} k_{m j},}, \quad \mathbf{y}_{k+2 s+\sum_{m=1}^{s} \sum_{j=1}^{4} k_{m j}-1}\right)=0 ;
\end{array}\right\}
$$

where $\mathbf{f}^{(\sigma)}=\left(f_{1}^{(\sigma)}, f_{2}^{(\sigma)}, f_{3}^{(\sigma)}, f_{4}^{(\sigma)}\right)^{T}$ is relative to governing equations of mapping $P_{\sigma} \quad(\sigma \in$ $\{1,2, \ldots, 6\})$. The periodicity of the period-1 motion per $N$-periods requires

$$
\mathbf{y}_{k+2 s+\sum_{m=1}^{s} \sum_{j=1}^{4} k_{m j}}=\mathbf{y}_{k}
$$

or

$$
\left.\begin{array}{rl}
x_{k+2 s+\sum_{m=1}^{s} \sum_{j=1}^{4} k_{m j}}^{(i)} & =x_{k}^{(i)}, \\
\dot{x}_{k+2 s+\sum_{m=1}^{s} \sum_{j=1}^{4} k_{m j}}^{(i)} & =\dot{x}_{k}^{(i)}, \\
\dot{x}_{k+2 s+\sum_{m=1}^{(\bar{i})} \sum_{j=1}^{4} k_{m j}}^{s} & =\dot{x}_{k}^{(\bar{i})}, \\
\Omega t_{k+2 s+\sum_{m=1}^{s} \sum_{j=1}^{4} k_{m j}} & \equiv \Omega t_{k}+2 N \pi .
\end{array}\right\}
$$

Solving Eqs. (41) and (42) generates the switching sets for periodic motions. Once the analytical prediction of any periodic motion is obtained, the corresponding stability and bifurcation analysis can be completed.

The local stability and bifurcation for such a period-1 motion is determined through the corresponding Jacobian matrix of the Poincaré mapping. From Eq. (40), the Jacobian matrix is computed by the chain rule, i.e.

$$
\begin{aligned}
D P= & D P_{\left(65^{k_{n 4}} 4^{k_{n 3}} 31^{k_{n 2}} 2^{k_{n 1}}\right) \cdots\left(65^{k_{14}} 4^{k_{13}} 31^{k_{12} 2^{k_{11}}}\right)} \\
= & \prod_{m=1}^{n}\left(D P_{6} \cdot D P_{5}^{k_{m 4}} \cdot D P_{4}^{k_{m 3}} \cdot D P_{3}\right. \\
& \left.\cdot D P_{1}^{k_{m 2}} \cdot D P_{1}^{k_{m 1}}\right)
\end{aligned}
$$

where

$$
D P_{\lambda}=\left[\begin{array}{cccc}
\frac{\partial t_{\nu+1}}{\partial t_{v}} & \frac{\partial t_{\nu+1}^{(i)}}{\partial x_{\nu}^{(i)}} & \frac{\partial t_{\nu+1}}{\partial y_{\nu}^{(i)}} & \frac{\partial t_{\nu+1}}{\partial y_{\nu}^{(\bar{i})}} \\
\frac{\partial x_{\nu+1}^{(i)}}{\partial t_{v}} & \frac{\partial x_{\nu+1}^{(i)}}{\partial x_{\nu}^{(i)}} & \frac{\partial x_{\nu+1}^{(i)}}{\partial y_{\nu}^{(i)}} & \frac{\partial x_{\nu+1}^{(i)}}{\partial y_{\nu}^{(\bar{i})}} \\
\frac{\partial y_{\nu+1}^{(i)}}{\partial t_{v}} & \frac{\partial y_{\nu+1}^{(i)}}{\partial x_{\nu}^{(i)}} & \frac{\partial y_{\nu+1}^{(i)}}{\partial y_{\nu}^{(i)}} & \frac{\partial y_{\nu+1}^{(i)}}{\partial y_{\nu}^{(\bar{i})}} \\
\frac{\partial y_{\nu+1}^{(\bar{i})}}{\partial t_{v}} & \frac{\partial y_{\nu+1}^{(\bar{i})}}{\partial x_{\nu}^{(i)}} & \frac{\partial y_{\nu+1}^{(\bar{i})}}{\partial y_{\nu}^{(i)}} & \frac{\partial y_{\nu+1}^{(\bar{i})}}{\partial y_{\nu}^{(\bar{i})}}
\end{array}\right]
$$

for $\nu=k, k+1, \ldots, k+2 s+\Sigma_{m=1}^{s} \Sigma_{j=1}^{4} k_{m j}-1$, and all the Jacobian matrix components can be computed through Eq. (41). The variational equation for a set of switching points $\left\{\mathbf{y}_{k}^{*}, \ldots, \mathbf{y}_{k+2 s+\Sigma_{m=1}^{*} \Sigma_{j=1}^{4} k_{m j}-1}\right\}$ is

$$
\Delta \mathbf{y}_{k+2 s+\sum_{m=1}^{s} \sum_{j=1}^{4} k_{m j}}=D P\left(\mathbf{y}_{k}^{*}\right) \Delta \mathbf{y}_{k}
$$




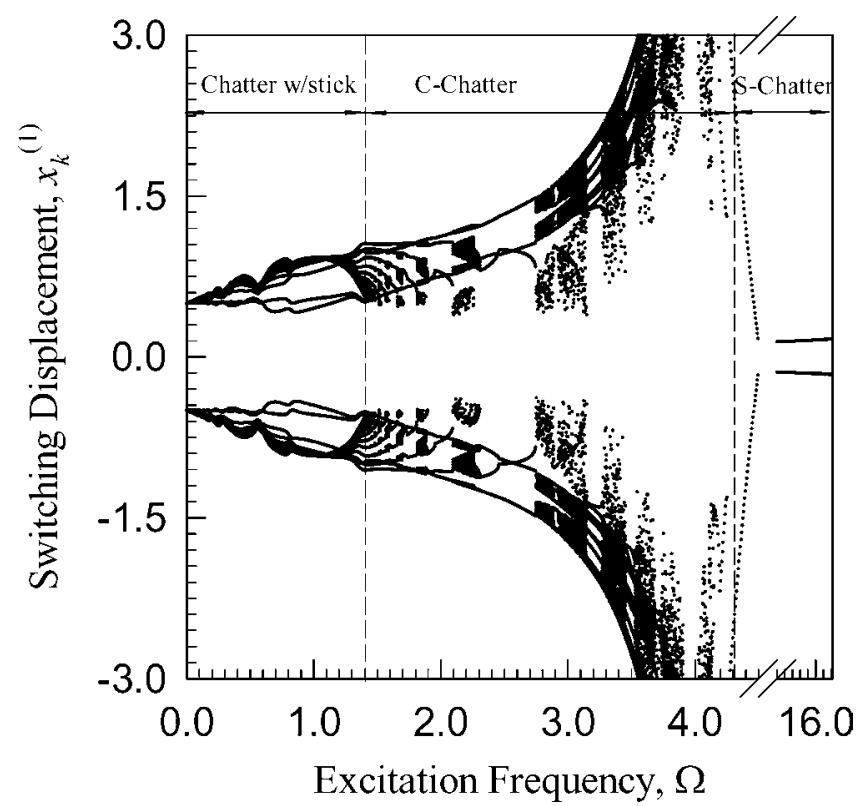

(a)

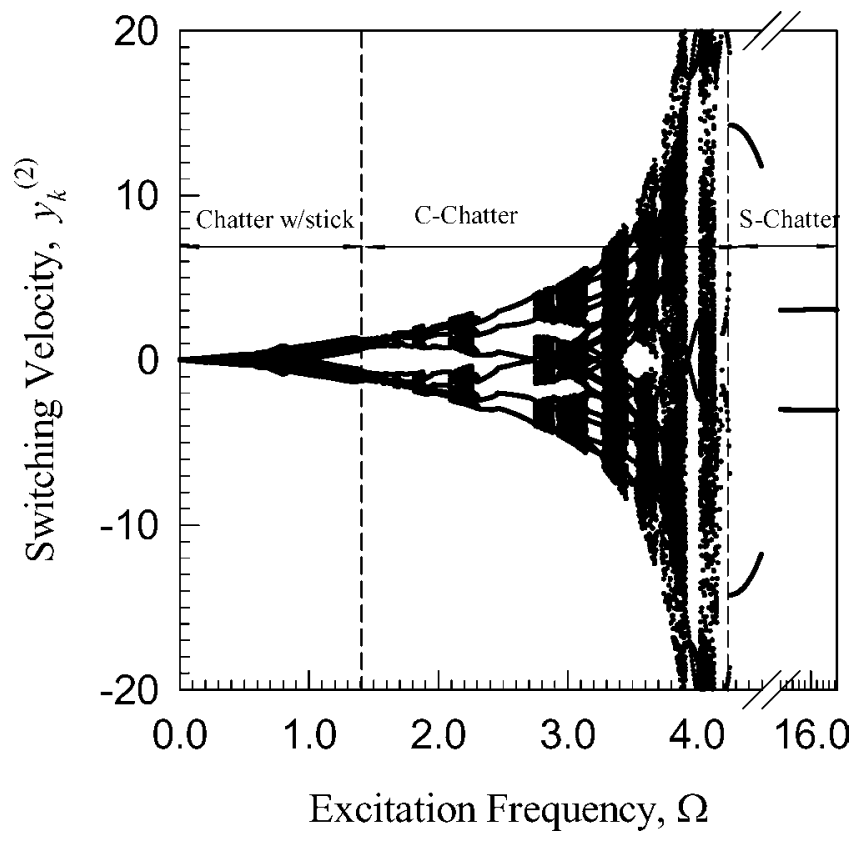

(c)

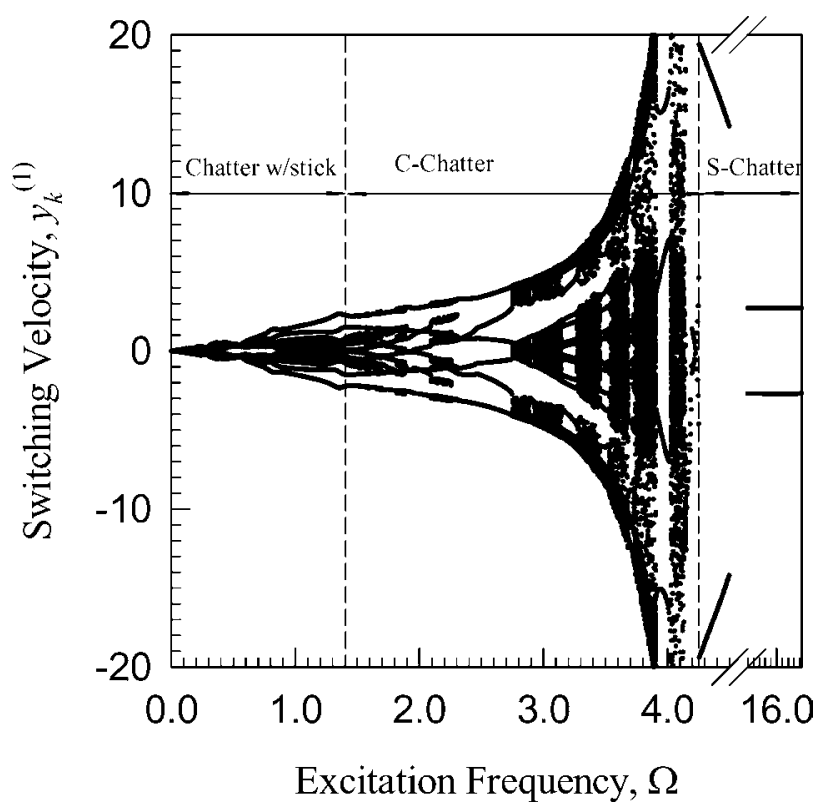

(b)

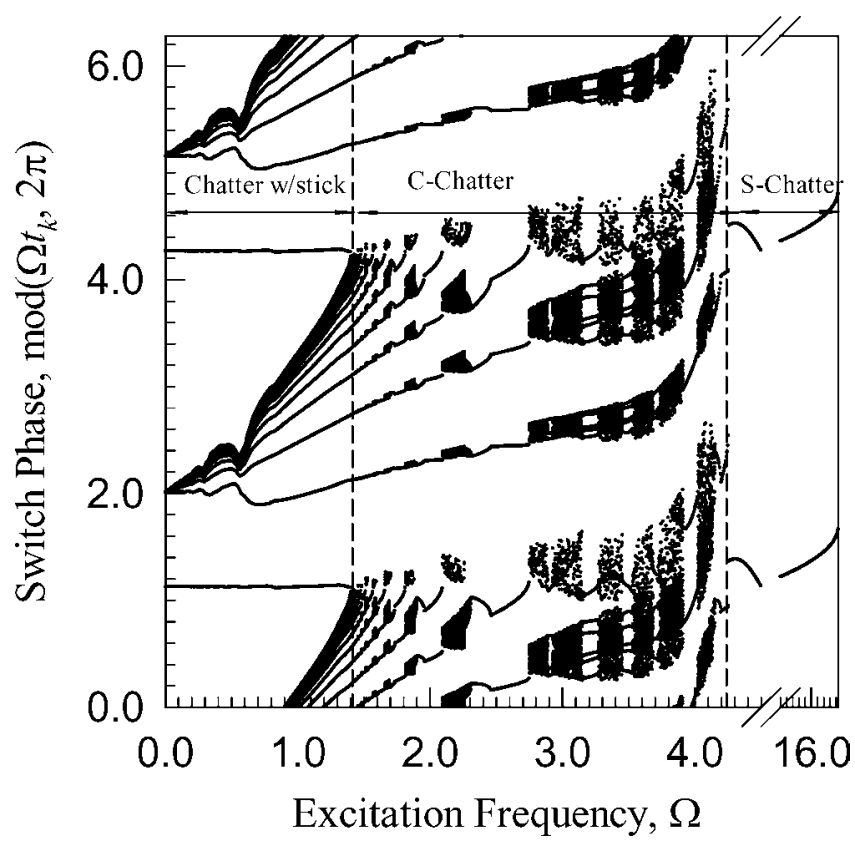

(d)

Fig. 5. Bifurcation scenario: (a) switching displacement of the first gear, (b) switching velocity of the first gear, (c) switching velocity of the second gear, and (d) switching phase $\left(m_{1}=2, m_{2}=1, r_{1}=r_{2}=0.6, k_{1}=30, k_{2}=20, Q_{0}=30, d=1.0\right.$ and $e=0.7$ ). 
Table 1. The summary of excitation frequency for impacting chatter with stick $\left(m_{1}=2\right.$, $m_{2}=1, r_{1}=r_{2}=0.6, k_{1}=30, k_{2}=20$, $Q_{0}=35, d=1.0$ and $\left.e=0.7\right)$.

\begin{tabular}{lcc}
\hline \multicolumn{2}{c}{ Mapping Structure } & Excitation Frequency \\
\hline$P_{62^{26} 135^{26} 4}$ & $P(T)$ & $(1.382,1.397)$ \\
$P_{62^{27} 135^{27} 4}$ & $P(T)$ & $(1.264,1.382)$ \\
$P_{62^{28} 135^{28} 4}$ & $P(T)$ & $(0.908,1.264)$ \\
$P_{62^{27} 135^{27} 4}$ & $P(T)$ & $(0.680,0.908)$ \\
$P_{62^{26} 135^{26} 4}$ & $P(T)$ & $(0.635,0.680)$ \\
$P_{62^{25} 135^{25} 4}$ & $P(T)$ & $(0.613,0.635)$ \\
$P_{62^{24}} 135^{24} 4$ & $P(T)$ & $(0.455,0.613)$ \\
$P_{62^{25}} 135^{25} 4$ & $P(T)$ & $(0.346,0.455)$ \\
$P_{62^{24}} 135^{24} 4$ & $P(T)$ & $(0.317,0.346)$ \\
$P_{62^{23}} 135^{23} 4$ & $P(T)$ & $(0.216,0.317)$ \\
$P_{62^{k}} 135^{k} 4$ & $P(T)$ & $(0.000,0.216)$ \\
\hline
\end{tabular}

The eigenvalues are computed by

$$
|D P-\lambda \mathrm{I}|=0 .
$$

Because $D P$ is a $4 \times 4$ matrix, there are four eigenvalues. If four eigenvalues lie inside the unit circle, then the period-1 motion is stable. If one of them lies outside the unit circle, the periodic motion is unstable. Namely, the stable, periodic motion requires the eigenvalues to be

$$
\left|\lambda_{j}\right|<1 \quad(j=1,2,3,4) .
$$

If the magnitude of one of the eigenvalues is greater than one,

$$
\left|\lambda_{j}\right|>1 \quad(j \in\{1,2,3,4\})
$$

the periodic motion is unstable.

For $\left|\lambda_{j}\right|<1(j=3,4)$ and real $\lambda_{j}(j=1,2)$,

(i) if

$$
\begin{gathered}
\max \left\{\lambda_{j}, j=1,2\right\}=1, \\
\min \left\{\lambda_{j}, j=1,2\right\} \in(-1,1),
\end{gathered}
$$

then the saddle-node (SN) bifurcation occurs;

(ii) if

$$
\begin{gathered}
\min \left\{\lambda_{j}, j=1,2\right\}=-1, \\
\max \left\{\lambda_{j}, j=1,2\right\} \in(-1,1),
\end{gathered}
$$

then the period-doubling bifurcation occurs.

For $\left|\lambda_{j}\right|<1(j=3,4)$ and complex $\lambda_{j}(j=$ $1,2)$,

$$
\left|\lambda_{j}\right|=1 \quad(j=1,2),
$$

then the Neimark bifurcation occurs.

Using the mapping structure in Eq. (32), all

\begin{tabular}{|c|c|c|}
\hline \multicolumn{2}{|l|}{ Mapping Structure } & Excitation Frequency \\
\hline$P_{63}$ & $P(T)$ & $(4.159,16.433)$ \\
\hline$P_{6532}$ & $P(T)$ & $(4.155,4.252)$ \\
\hline$P_{(6532)^{2}}$ & $P(2 T)$ & $(4.150,4.155)$ \\
\hline & chaos & $(4.10,4.150)$ \\
\hline$P_{(6553265)(3226532)}$ & $P(3 T)$ & $(4.095,4.100)(3.683,3.724)$ \\
\hline$P_{((6553265)(3226532))^{2}}$ & $P(6 T)$ & $(4.094,4.095)(3.725,3.727)$ \\
\hline$P_{((6553265)(3226232))^{4}}$ & $P(12 T)$ & $(3.727,3.728)$ \\
\hline$P_{655322}$ or $P_{322655}$ & $P(T)$ & $(3.909,4.022)$ \\
\hline & chaos & $(3.728,3.909)$ \\
\hline$P_{((65532653265)(32265326532))^{4}}$ & $P(20 T)$ & $(3.528,3.724)$ \\
\hline$P_{((65532653265)(32265326532))^{2}}$ & $P(10 T)$ & $(3.524,3.528)$ \\
\hline$P_{(65532653265)(32265326532)}$ & $P(5 T)$ & $(3.455,3.524)$ \\
\hline & chaos & $(3.267,3.455)$ \\
\hline$P_{(65532653265)(32265326532)}$ & $P(5 T)$ & $(3.150,3.267)$ \\
\hline & chaos & $(3.020,3.150)$ \\
\hline$P_{(65532)(65322)(6532)(6532)}$ & $P(4 T)$ & $(3.014,3.020)$ \\
\hline & chaos & $(2.925,3.014)$ \\
\hline$P_{(6553265)(3226532)}$ & $P(3 T)$ & $(2.889,2.925)$ \\
\hline & chaos & $(2.748,2.889)$ \\
\hline$P_{65^{2} 32^{2}}$ & $P(T)$ & $(2.309,2.748)$ \\
\hline$P_{65^{m}} 32^{m}(m=1,2, \ldots, 10)$ and chaos & $P(T)$ & $(1.378,2.309)$ \\
\hline
\end{tabular}
the periodic motions for the entire range of excitation frequency can be determined analytically by the corresponding governing equations similar to Eqs. (42) and (43). The mapping structure gives a set of nonlinear algebraic equations, which

Table 2. The summary of excitation frequency for impacting chatter $\left(m_{1}=2\right.$, $m_{2}=1, r_{1}=r_{2}=0.6, k_{1}=30, k_{2}=20, Q_{0}=35, d=1.0$ and $\left.e=0.7\right)$. 
can be solved by the Newton-Raphson method. To make the numerical computation convergent fast, the initial guess solutions should be chosen in the appropriate convergent domain. Once the first solution is obtained, the remaining solutions varying with parameters can be determined through the corresponding mapping structure. For the analytical prediction of periodic motions, the parameters $\left(m_{1}=2, m_{2}=1, r_{1}=r_{2}=0.6\right.$, $k_{1}=30, k_{2}=20, Q_{0}=30, d=1.0, e=0.7$ ) are used. The analytical prediction of the periodic motion of $P_{64^{2} 32^{2}}$ gives switching sets varying with excitation frequency, as shown in Fig. 6. Because of the page limitation, the eigenvalues varying with

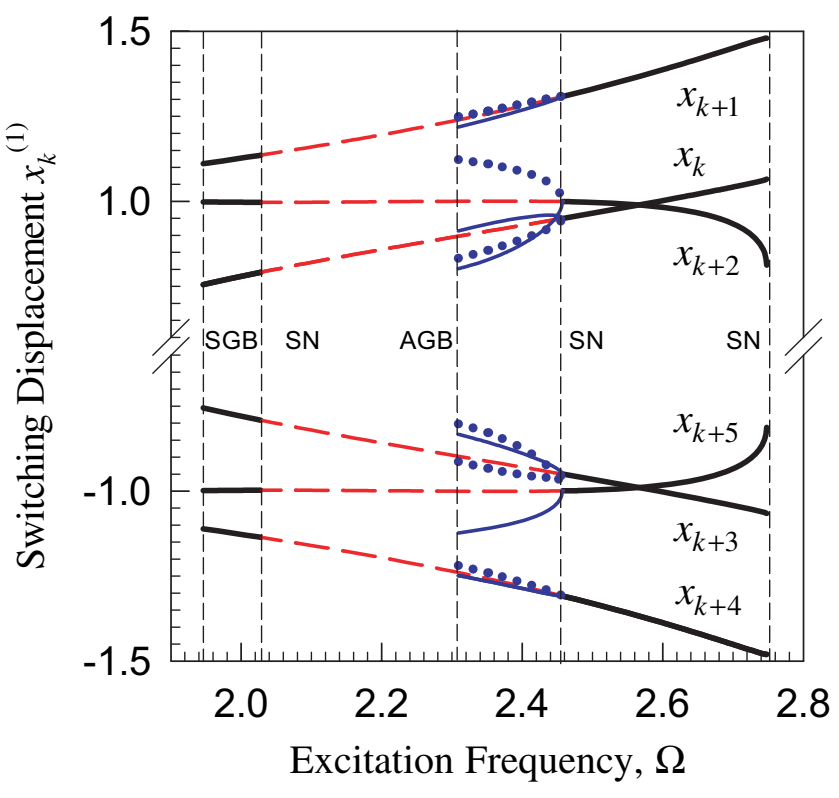

(a)

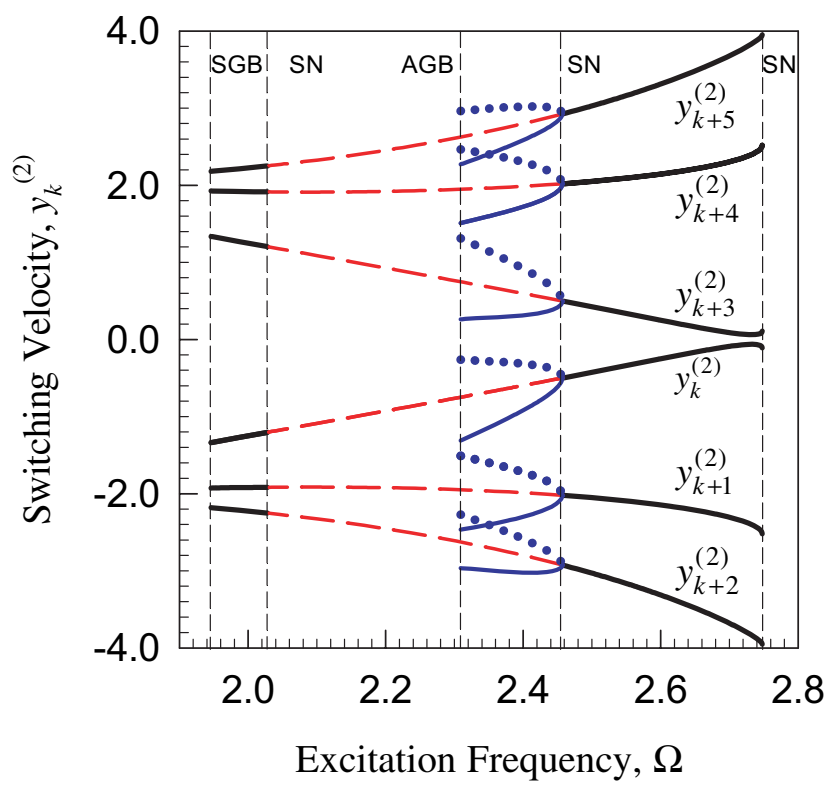

(c)

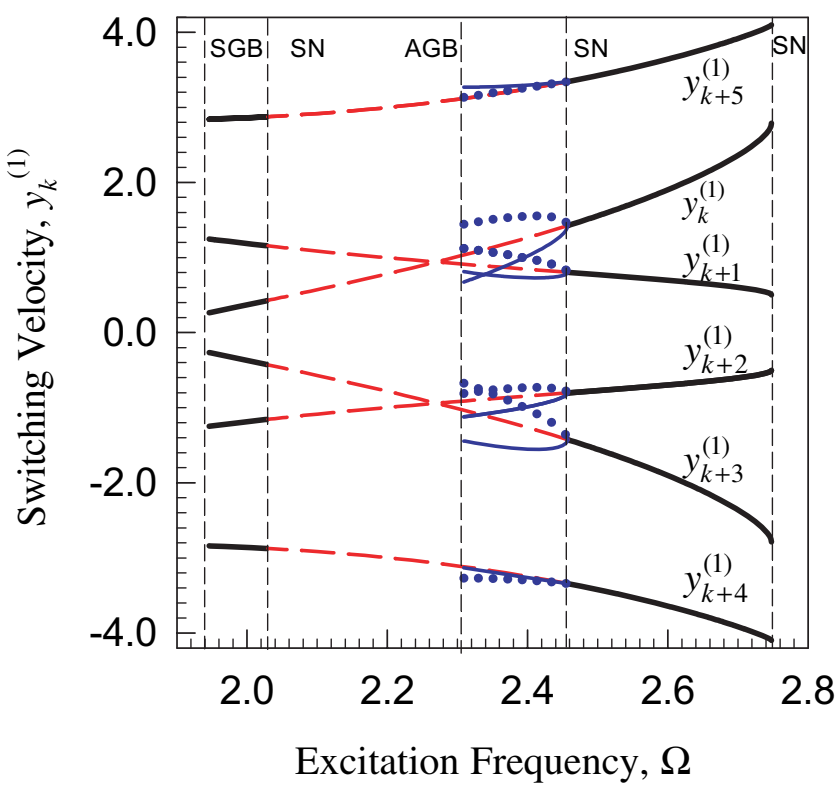

(b)

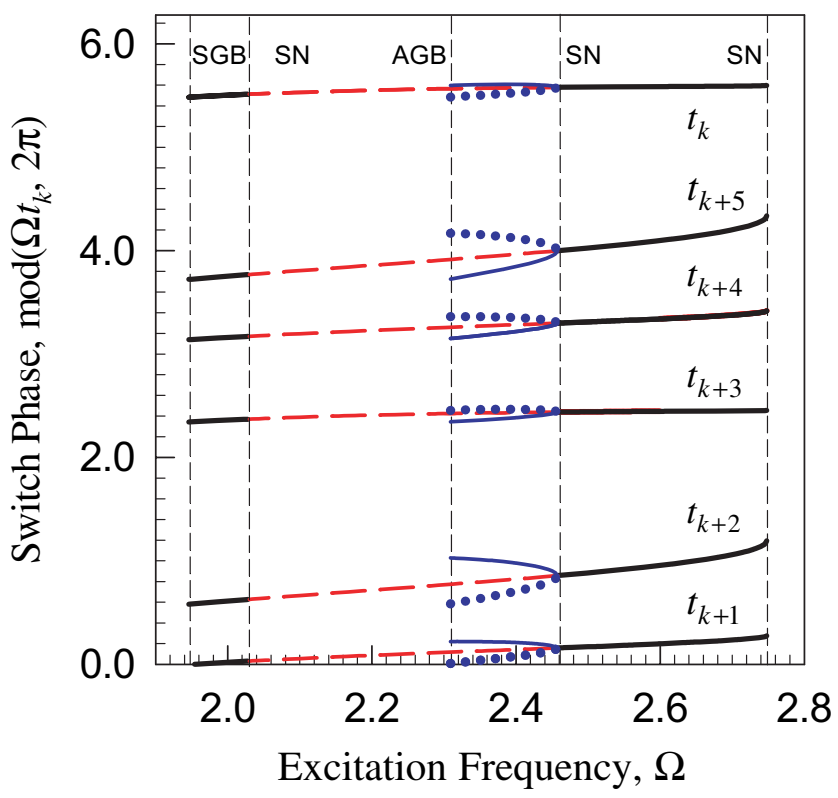

(d)

Fig. 6. The analytical prediction of a periodic motion of impacting chatter $P_{64^{2} 32^{2}}$ : (a) switching displacement and (b) switching velocity of the first gear, (c) switching velocity of the second gear, and (d) switching phase $\left(m_{1}=2, m_{2}=1, r_{1}=r_{2}=0.6\right.$, $k_{1}=30, k_{2}=20, Q_{0}=30, d=1.0$ and $\left.e=0.7\right)$. 
excitation frequency will not be plotted herein. In four plots, the switching displacement and velocity of the first gear are plotted in Figs. 6(a) and 6(b), and for the second gear, only the switching velocity of the second gear is presented in Fig. 6(c). At switching points, the switching displacement of the second gear can be obtained from the switching displacement of the first gear, i.e. $x_{k}^{(2)}=x_{k}^{(1)} \pm d / 2$. The corresponding switching phase is presented in Fig. 6(d). The switching sets are recorded before impact rather than after impact. The range for the stable, symmetric motion of $P_{64^{2} 32^{2}}$ is

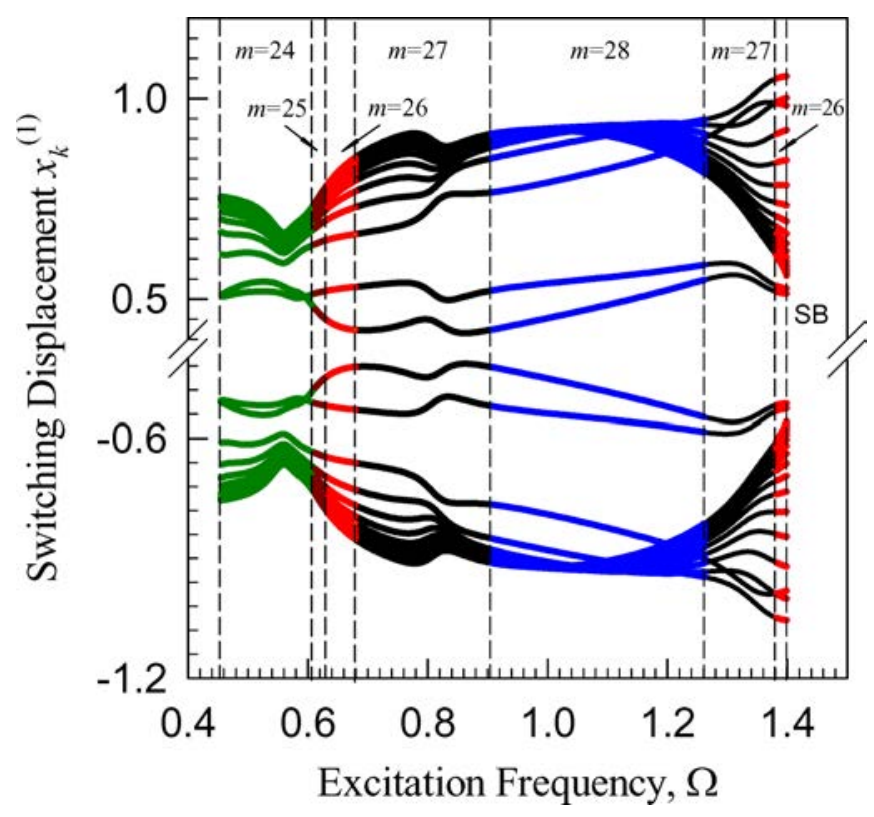

(a)

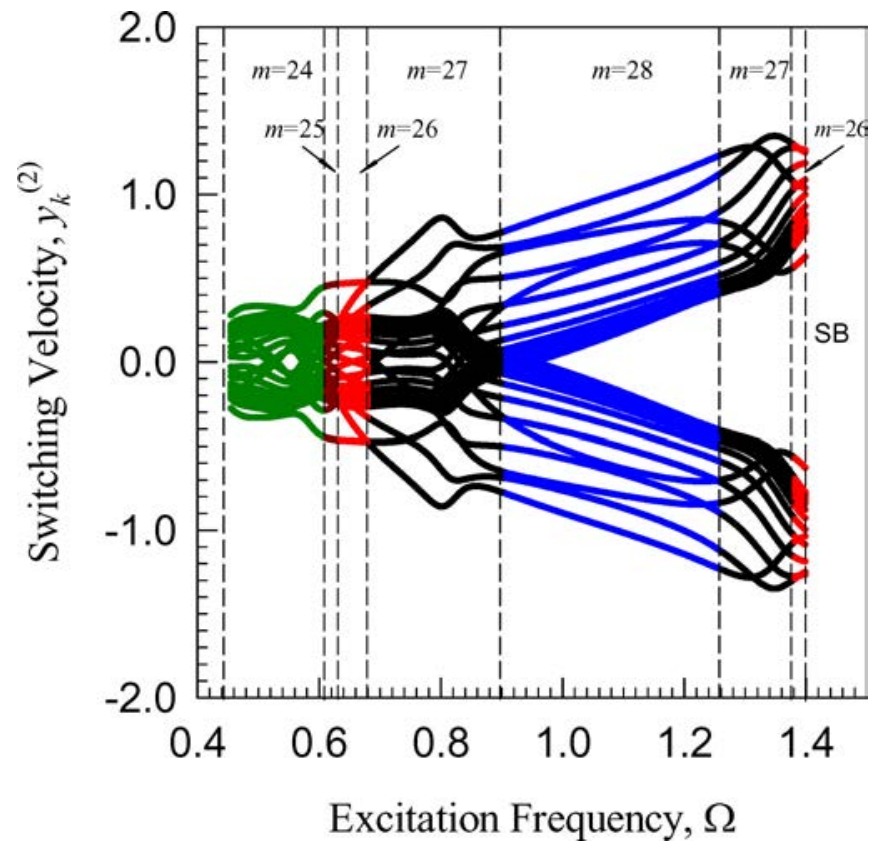

(c)

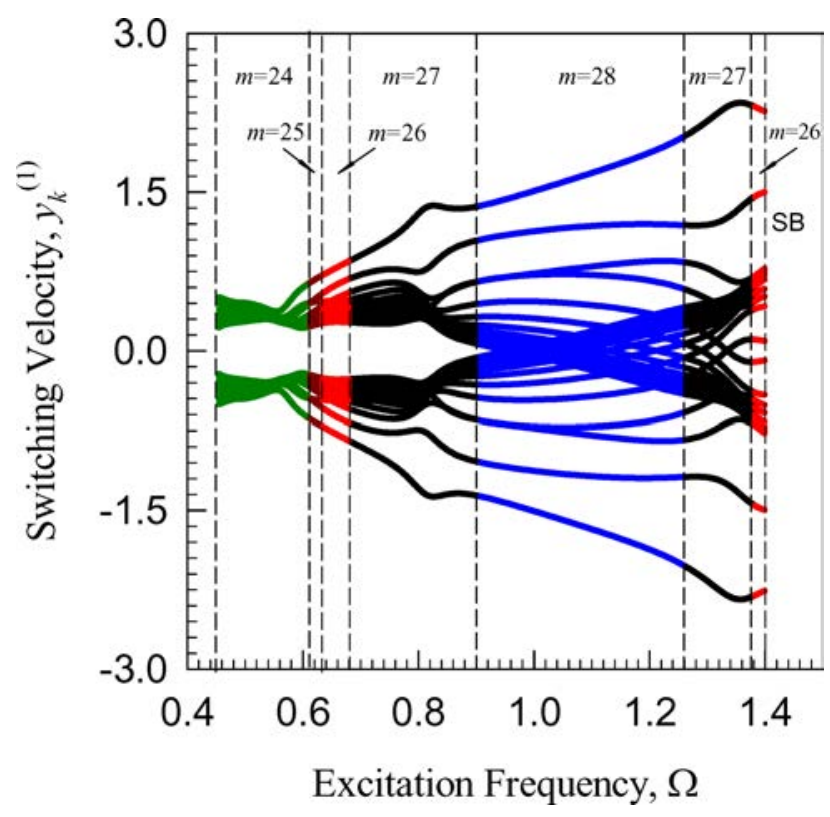

(b)

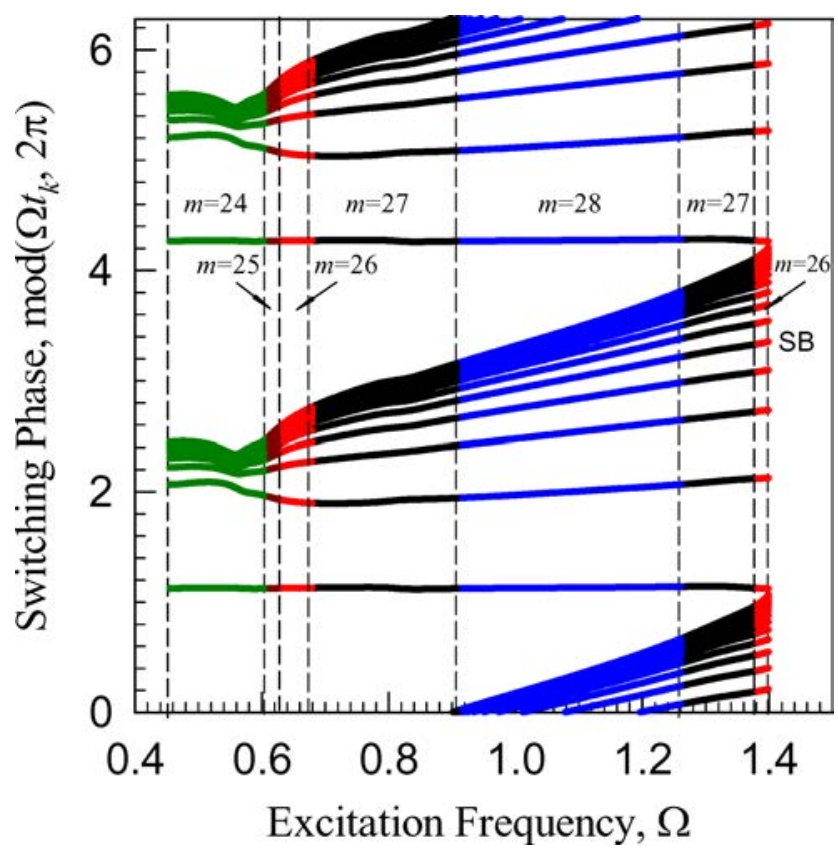

(d)

Fig. 7. The analytical prediction of a periodic motion of impacting chatter with stick $P_{645^{m}} 312^{m}(m=24,25, \ldots, 28)$ : (a) switching displacement and (b) switching velocity of the first gear, (c) switching velocity of the second gear, and (d) switching phase $\left(m_{1}=2, m_{2}=1, r_{1}=r_{2}=0.6, k_{1}=30, k_{2}=20, Q_{0}=30, d=1.0\right.$ and $\left.e=0.7\right)$. 
$\Omega \in\{(2.457,2.748),(1,946,2.028)\}$, which is represented by thick curves. The unstable symmetric periodic motion for such a mapping structure lies in $\Omega \in(2.028,2.457)$, depicted by the dashed curves. For the same mapping structure, the asymmetric, periodic motion exists in the range of $\Omega \in$ $(2.380,2.457)$, and there are two branches of solutions. The two branches of asymmetric solutions are presented through the dotted and thin curves. The saddle-node bifurcation of the symmetric, periodic motion occurs at $\Omega \approx 2.457$ and 2.748 and the saddle-node bifurcation of the asymmetric periodic motion is $\Omega \approx 2.457$. Such a saddle-node bifurcation causes the symmetry break of impacting chatter motion. The grazing bifurcations for the symmetric and asymmetric periodic motions are $\Omega \approx 2.380$ and 1.946. For the grazing periodic motion, the eigenvalues show the two periodic motions are stable, i.e. the eigenavalues of the grazing periodic motion lie in the unit circle. However, the grazing causes the mapping structure to be changed. Thus the motion relative to the old mapping structure disappears. In a similar fashion, all the other periodic motion of impacting chatter can be predicted analytically.

Using the same parameters, the analytical prediction of periodic motions with stick is given herein. The corresponding switching sets varying with excitation frequency are presented in Figs. $7(\mathrm{a})-7(\mathrm{~d})$ for the mapping structure of $P_{654^{m} 312^{m}}(m=24,25, \ldots, 28)$. Because the computational time is very consumed, the impacting chatters with stick are predicted only in the range of excitation frequency $\Omega \in(0.455,1.39859)$. The stick bifurcation (SB) occurs at $\Omega \approx 1.39859$. Once the stick motion appears, the impacting chatter with stick for $P_{645^{26} 312^{26}}$ are in the range of $\Omega \in(1.328,1.39859)$ and $(0.635,0.6789)$. The stick motion of $P_{654^{27} 312^{27}}$ lies in two regions of $\Omega \in$ $(1.264,1.328)$ and $(0.680,0.9068)$. The stick motion of $P_{645^{28} 312^{28}}$ is in $\Omega \in(0.9068,1.264)$. The stick motions of $P_{645^{25} 312^{25}}$ and $P_{645^{24} 312^{24}}$ are in $\Omega \in$ $(0.612,0.6789)$ and $(0.455,0.612)$, respectively. In stick motion computation, the computational criterion (i.e. $\left|\dot{x}_{k}^{(1)}-\dot{x}_{k}^{(2)}\right|<10^{-6}$ ) was embedded. If the tolerance is set much smaller, the analytical and numerical predictions cannot be matched sometimes. Such a problem may be caused by the computational error. For a higher accuracy, a better algorithm should be developed. In the other ranges of the stick motion, the analytical prediction can be carried out analytically.

\section{Numerical Simulations}

From the analytical prediction, periodic motions for impacting chatter with/without stick will be simulated numerically. All the responses of the first and second gears are represented by the thick and thin curves, respectively. The left and right sides of the second gear are presented by the intermediate thick curves. The switching points before and after impacts are represented by circles. The hollow and gray-filled circles are for the first and second gears, accordingly. The switching points for stick are represented by the large filled circles.

For system parameters $\left(m_{1}=2, m_{2}=1, r_{1}=\right.$ $r_{2}=0.6, k_{1}=30, k_{2}=20, Q_{0}=30, d=1.0$ and $e=0.7)$, consider the excitation frequency $\Omega=2.72$. The analytical prediction gives the initial condition (i.e. $t_{0} \approx 2.0559169, x_{0}^{(1)} \approx 1.0505790$, $x_{0}^{(2)} \approx 0.5505790, y_{0}^{(1)} \approx 1.68706528$ and $y_{0}^{(2)} \approx$ 4.5219068 ) for the stable periodic impacting chatter of $P_{64}{ }^{2} 32^{2}$. The displacement and velocity timehistories, trajectories of the first and second gears in phase plane are presented in Figs. 8(a)-8(d). The displacement responses of two gears are presented in Fig. 8(a). The motion starts at the right side (Rside) of the second gear. The first gear with the second gear has two impacts. Afterwards, the two gears have a free-flying motion. Once the first gear arrives to the left side (L-side) of the second gear, the next two impacts between the two gears occurs. After these two impacts, the two gears have another freeflying motion, and the two gears meet on the right side of the second gear. Such a motion process will repeat and form a periodic motion. Due to impacts between two gears on the left and right sides, the impacting velocities become discontinuous. Thus, the velocity time-histories for two gears are presented in Fig. 8(b). Such discontinuity is observed at the impact locations. After the right side impact, the first gear velocity is less than the second gear velocity (i.e. $y_{k}^{(1)}<y_{k}^{(2)}$ ), and vice versa for the left side. The motions of two gears are separated owing to $y_{k}^{(1)} \neq y_{k}^{(2)}$ before and after impacts. To observe the periodic behaviors of the two gears, the trajectories of such a periodic motion in phase plane for the first and second gears are presented in Figs. 8(c) and 8(d), accordingly. The mapping structure of $P_{64^{2} 32^{2}}$ is observed via the periodic loop. In Fig. 8(d), the absolute displacement of the central location of the second gear is employed for illustration, instead of the left and right sides of the second gear. However, the left and right tooth locations of 


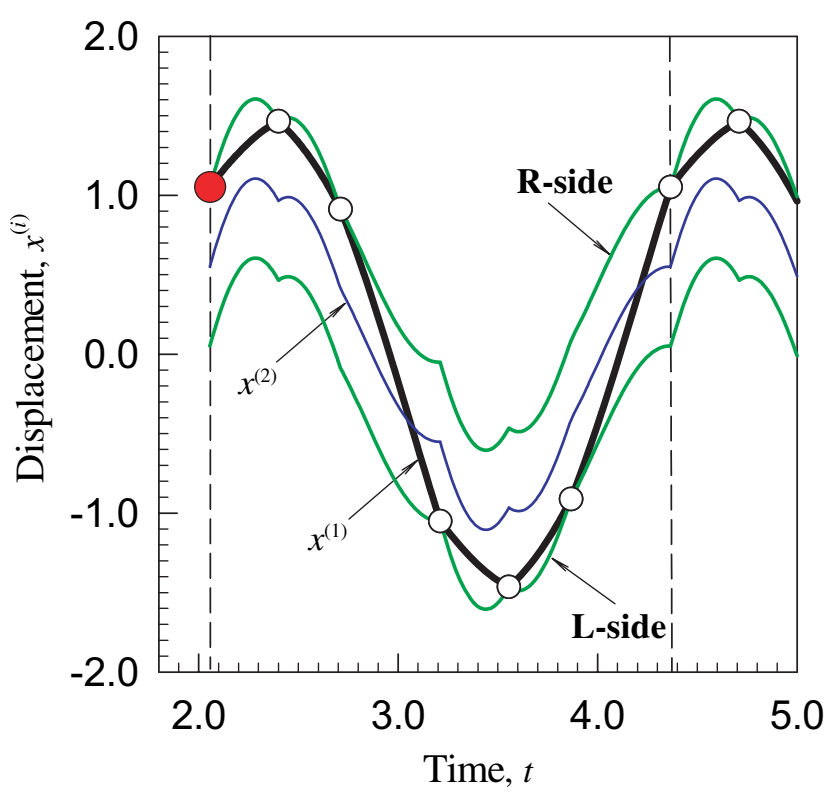

(a)

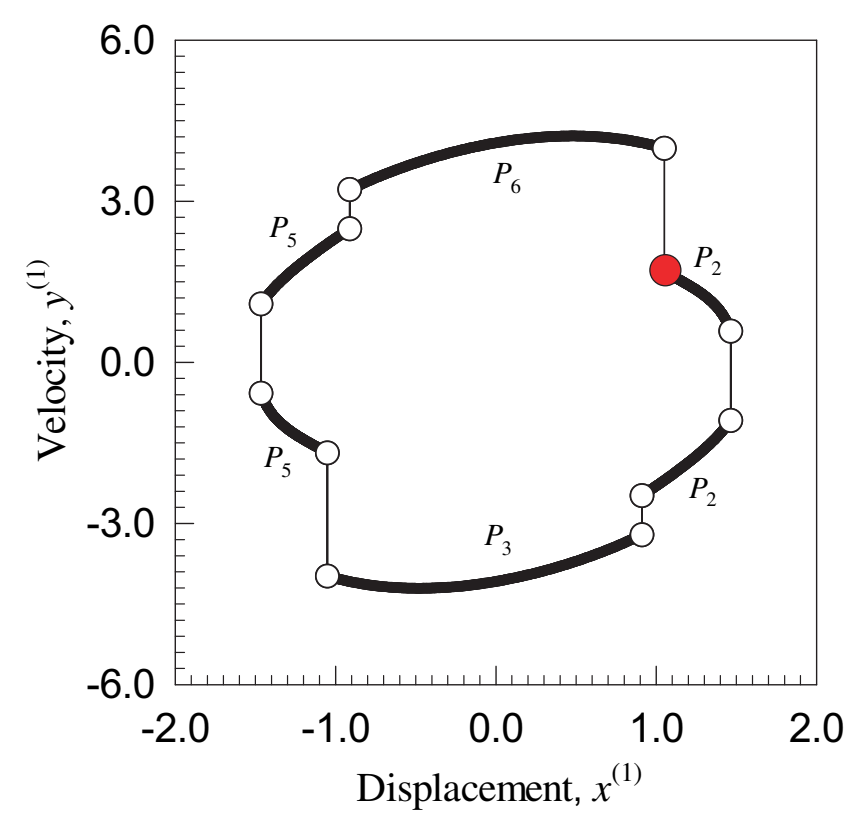

(c)

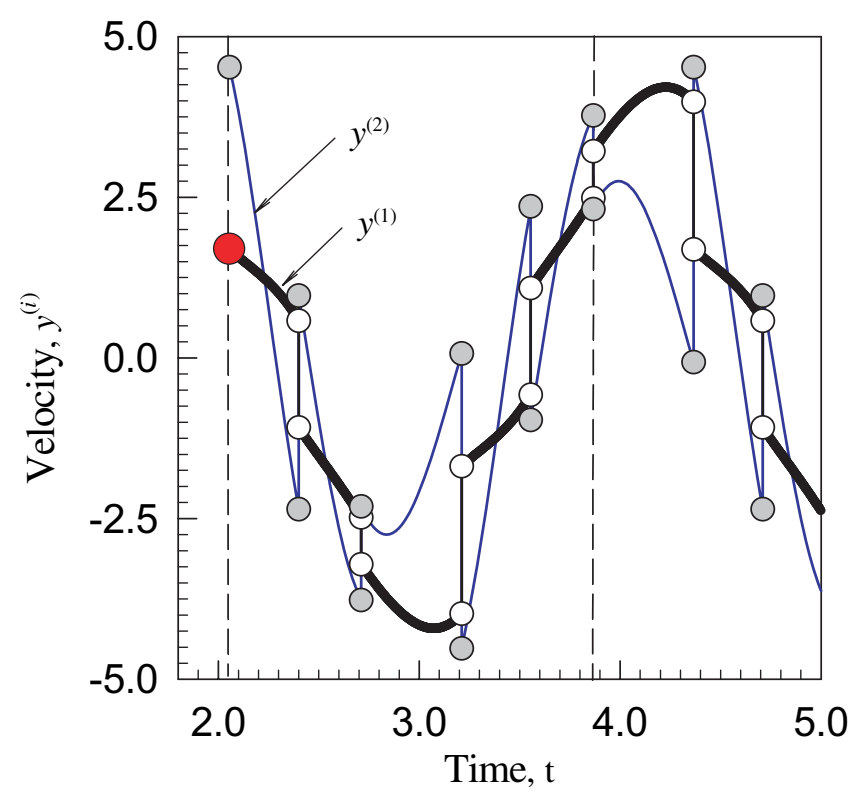

(b)

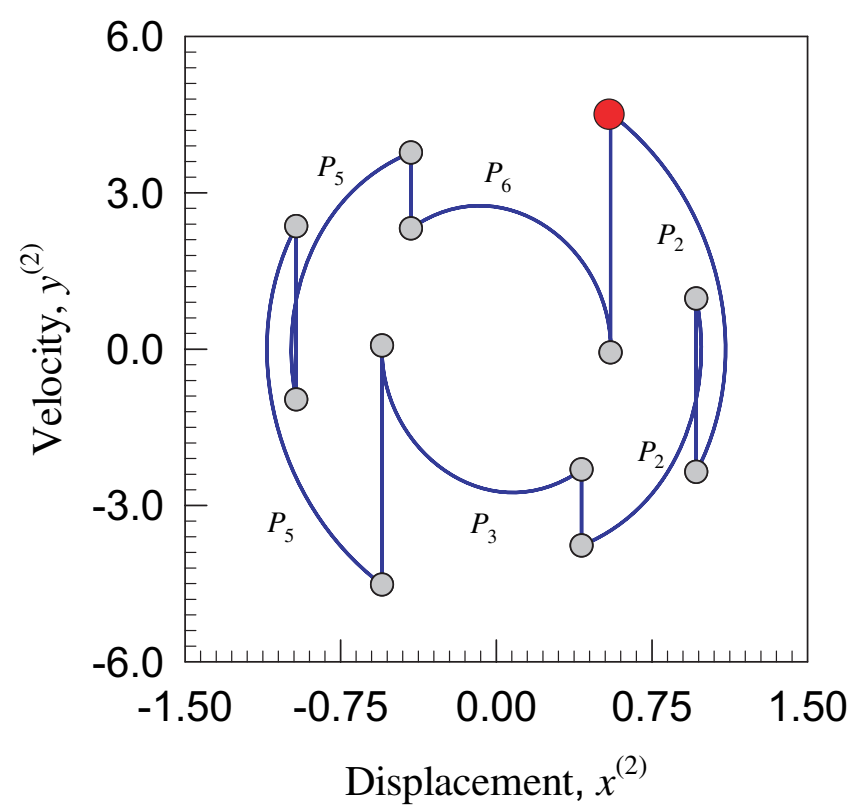

(d)

Fig. 8. The periodic motion of impacting chatter $P_{65^{2} 32^{2}}$ : (a) displacement time-history and (b) velocity time-history, (c) trajectories of the first gear, $(\mathrm{d})$ trajectories of the second gear in phase plane $\left(m_{1}=2, m_{2}=1, r_{1}=r_{2}=0.6, k_{1}=30, k_{2}=20\right.$, $Q_{0}=30, d=1.0, e=0.7$ and $\left.\Omega=2.72\right)$. The initial condition is $t_{0} \approx 2.0559169, x_{0}^{(1)} \approx 1.0505790, x_{0}^{(2)} \approx 0.5505790$, $y_{0}^{(1)} \approx 1.68706528$ and $y_{0}^{(2)} \approx 4.5219068$.

the second gear can be obtained by adding the two offsets.

To understand such impacting chatter in the gear transmission system, the relative force responses should be discussed. After the first rightside impact, to make the repeated impact occur, the relative force should be greater than zero (i.e. $\left.g_{\alpha}^{(1)}\left(t_{k}\right)>0\right)$. Since the absolute velocity relation $\left(y_{k}^{(1)}<y_{k}^{(2)}\right)$ holds just after impact, it means the relative velocity is less than zero $\left(\dot{z}_{k}^{(1)}=y_{k}^{(1)}-\right.$ $\left.y_{k}^{(2)}<0\right)$. Thus, just after the impact, the relative 
displacement is less than half a gap (i.e. $z_{k}^{(1)}=$ $\left.x_{k}^{(1)}-x_{k}^{(2)}<d / 2\right)$. To allow the first gear to catch up with the second one, the relative force should be greater than zero (i.e. $g_{\alpha}^{(1)}\left(t_{k}\right)>0$ ) before the next impact. Otherwise, the next impact on the righthand side will not happen. In a similar fashion, just after the first impact on the left-side the relative force should be less than zero (i.e. $g_{\alpha}^{(1)}\left(t_{k}\right)<0$ ) to achieve the second impact. From Figs. 9(a) and 9 (b), such a relation is observed. Based on the perspective of the second gear, the relative force criterion of the periodic motion is described through $z^{(2)}=x^{(2)}-x^{(1)}=-z^{(1)}$, and the relative velocity and force for the second gear should be opposite to the first gear. So the relative force for the second gear is presented in Figs. 9(a) and 9(b) as well.

The impacting chatter with stick in the gear transmission system is very interesting. Such a periodic motion will be discussed through the mapping structure of $P_{5}{ }^{25} 312^{25} 64$. The impacting chatter with stick relative to $P_{5^{25}} 312^{25} 64$ is equivalent to the one relative to $P_{645^{25} 312^{25}}$. Just the starting points are from different switching points. As before, the same parameters will be adopted for this illustration with $\Omega=0.5$. From the analytical prediction, the initial condition is selected from the switching point $t_{0} \approx$
4.8888960, $x_{0}^{(1)} \approx-0.7387947, x_{0}^{(2)} \approx-0.2387947$, and $y_{0}^{(1)}=y_{0}^{(2)} \approx-0.2754161$. From such an initial condition, the stick motion will appear on the left side of the second gear. After the stick vanishes, the first gear moves to the right side of the second gear through the free-flying motion. Because of different velocities of the two gears, the impacting chatter occurs before the stick appears. For this motion, once $y_{k}^{(1)}=y_{k}^{(2)}$, the stick motion will be observed. In Fig. 10, the displacement, velocity and trajectories in phase planes are presented. The shaded area is used for stick motion, and the Letters "L" and " $\mathrm{R}$ " represent the left-side and right-side of the second gear, respectively. In Fig. 10(a), the displacement responses of the two gears are presented. The stick motion on the left and right sides of the second gear are observed. The impacting chatter with 26 impacts on each side is observed. To further confirm the impacts, the velocity responses for the two gears are presented in Fig. 10(b). For further illustration of the impacting chatter with stick, the trajectories of the first and second gears in phase plane are plotted in Figs. 10(c) and 10(d), respectively.

For a better understanding of impacting chatter with stick, the relative force and jerk for the two gears are illustrated in Fig. 11. The impacting

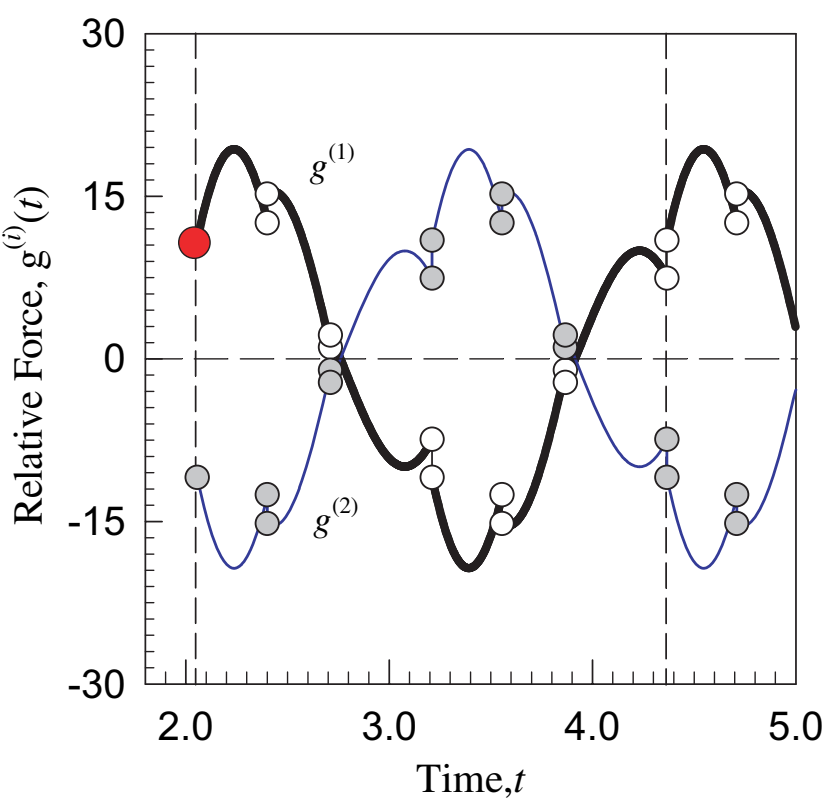

(a)

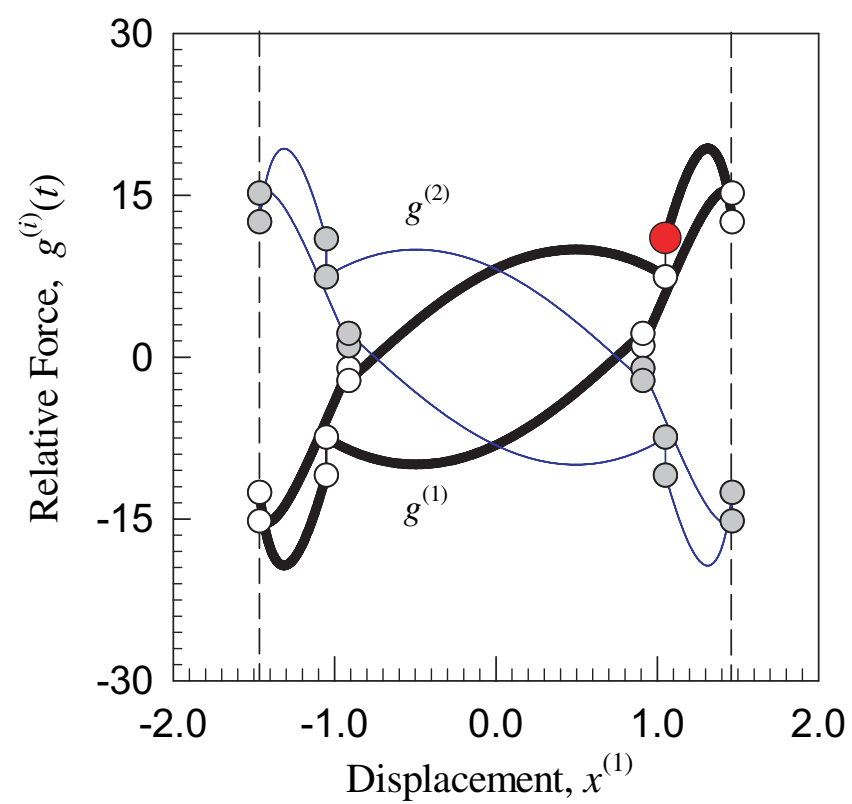

(b)

Fig. 9. Relative forces for the periodic motion of impacting chatter $P_{65^{2}} 32^{2}$ : (a) time-history and (b) distribution along the displacement of the first gear $\left(m_{1}=2, m_{2}=1, r_{1}=r_{2}=0.6, k_{1}=30, k_{2}=20, Q_{0}=30, d=1.0, e=0.7\right.$ and $\left.\Omega=2.72\right)$. The initial condition is $t_{0} \approx 2.0559169, x_{0}^{(1)} \approx 1.0505790, x_{0}^{(2)} \approx 0.5505790, y_{0}^{(1)} \approx 1.68706528$ and $y_{0}^{(2)} \approx 4.5219068$. 


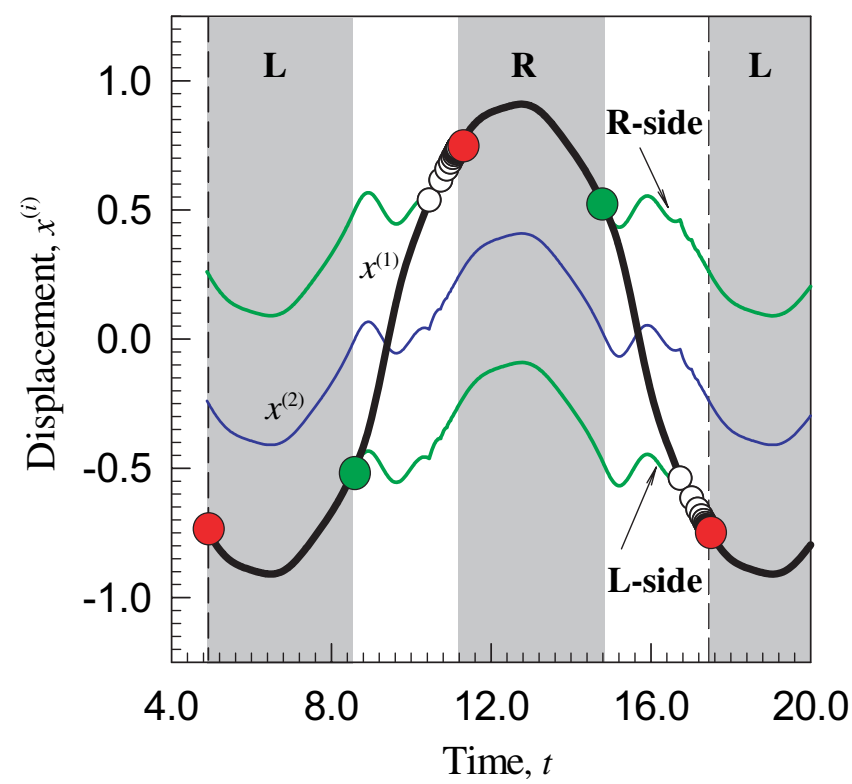

(a)

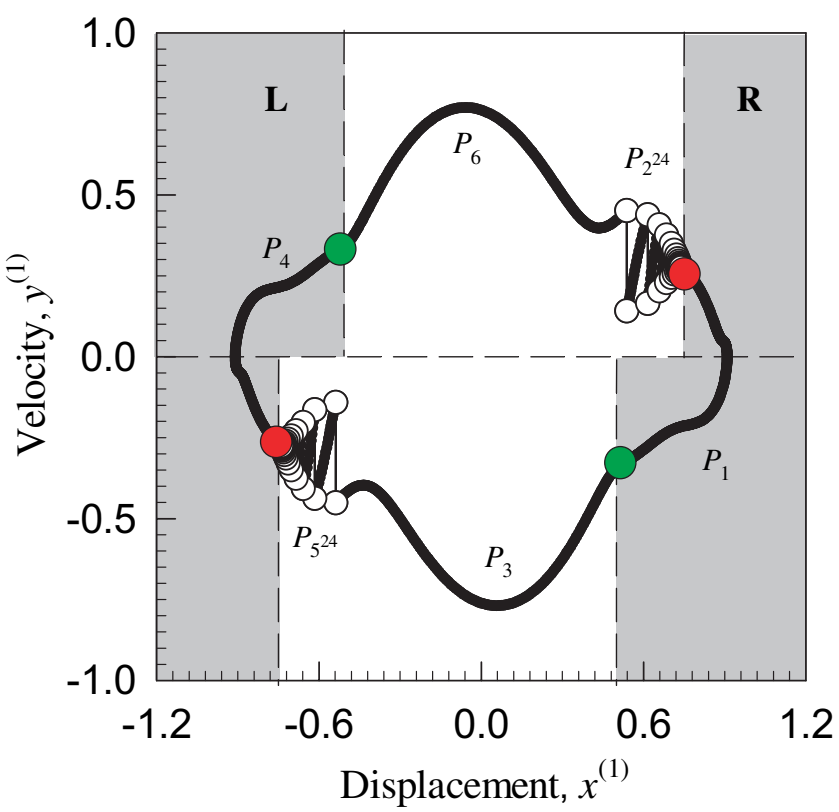

(c)

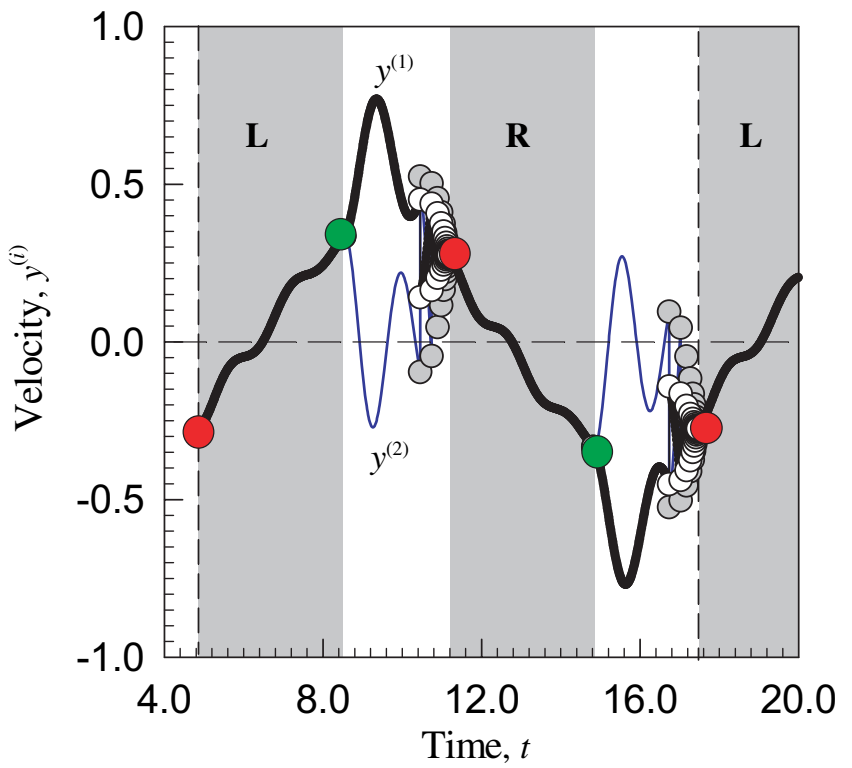

(b)

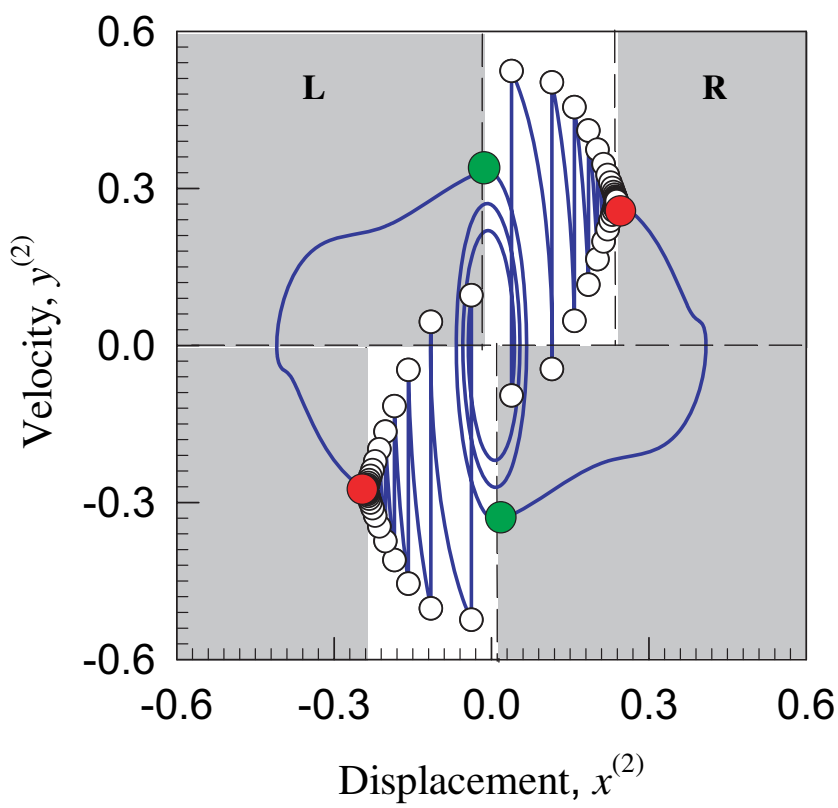

(d)

Fig. 10. The periodic motion of impacting chatter with stick $P_{645^{25}} 312^{25}$ : (a) displacement time-history and (b) velocity time-history, (c) trajectories of the first gear, (d) trajectories of the second gear in phase plane $\left(m_{1}=2, m_{2}=1, r_{1}=r_{2}=\right.$ $0.6, k_{1}=30, k_{2}=20, Q_{0}=30, d=1.0, e=0.7$ and $\left.\Omega=0.5\right)$. The initial condition is $t_{0} \approx 4.8888960, x_{0}^{(1)} \approx-0.7387947$, $x_{0}^{(2)} \approx-0.2387947$ and $y_{0}^{(1)}=y_{0}^{(2)} \approx-0.2754161$.

motion between the two gears was discussed in Fig. 9. Herein, the discussion focuses on the portion of the stick motion. On the switching boundary with $y_{k}^{(1)}=y_{k}^{(2)}$, the stick motion requires $g_{\alpha}^{(1)}\left(t_{k}\right)>0$ (or $g_{\alpha}^{(2)}\left(t_{k}\right)<0$ ) on the right-side for $\alpha=1,2$ and $g_{\alpha}^{(1)}\left(t_{k}\right)<0$ (or $g_{\alpha}^{(2)}\left(t_{k}\right)>0$ ) on the left-side for $\alpha=2,3$. Because the vector fields in different domains for such a discontinuous motion are different, the relative forces and jerks in the different domains are totally different (i.e. 


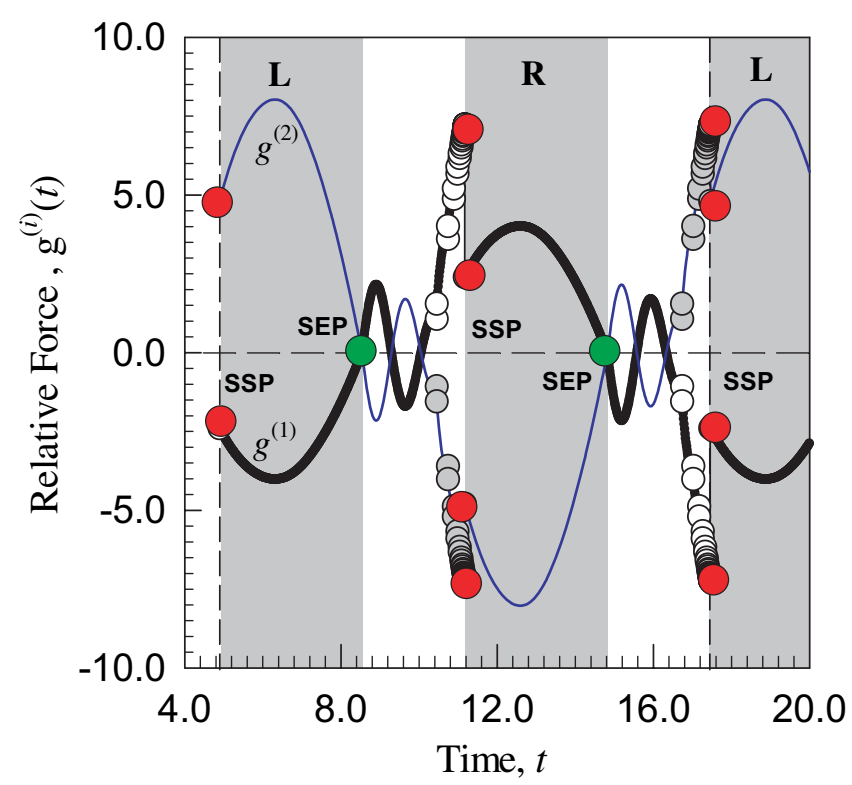

(a)

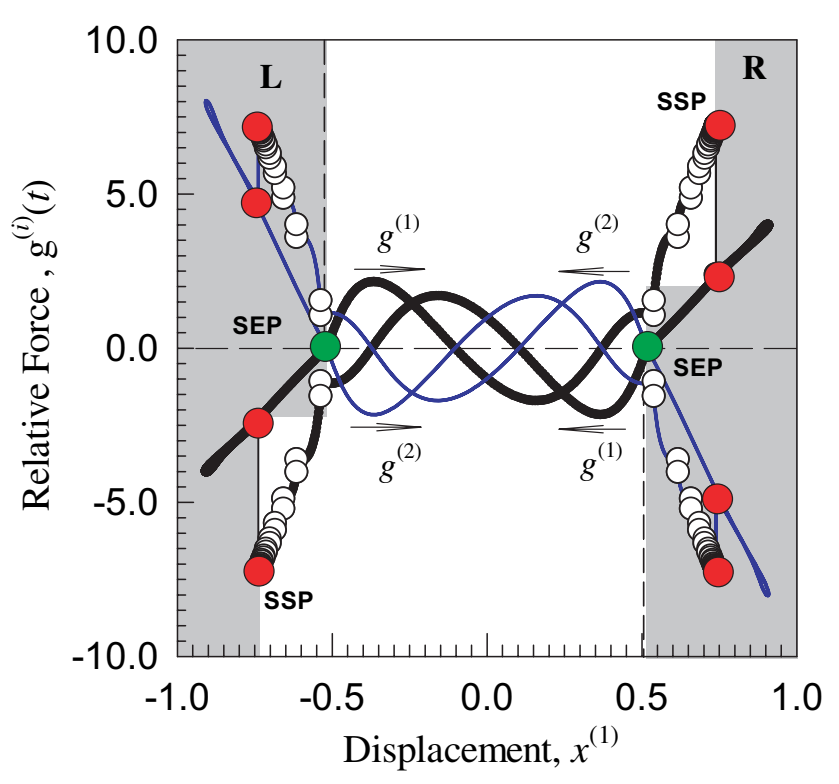

(c)

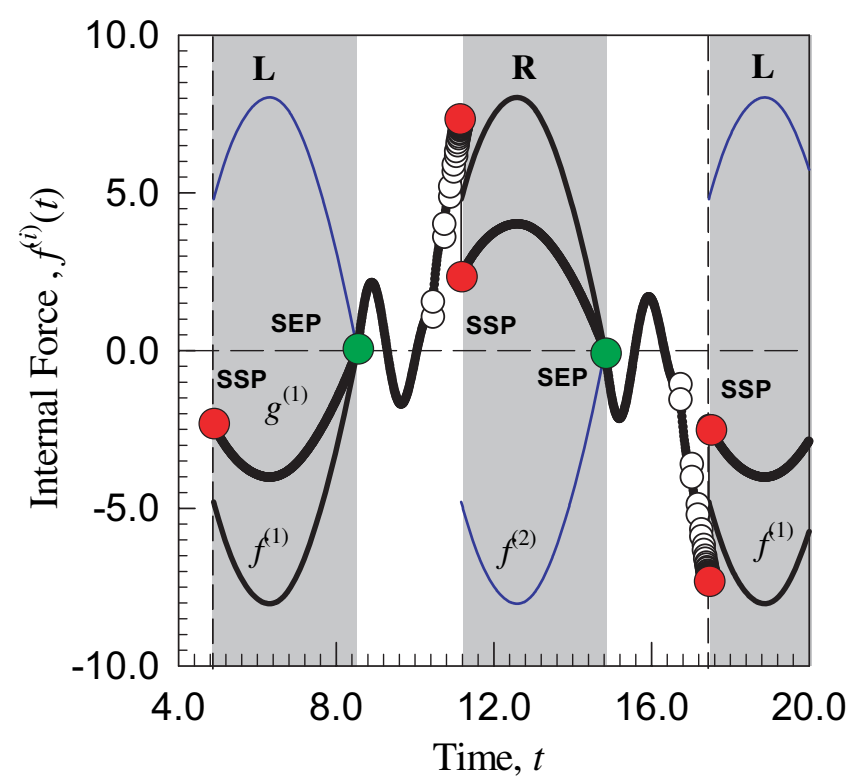

(b)

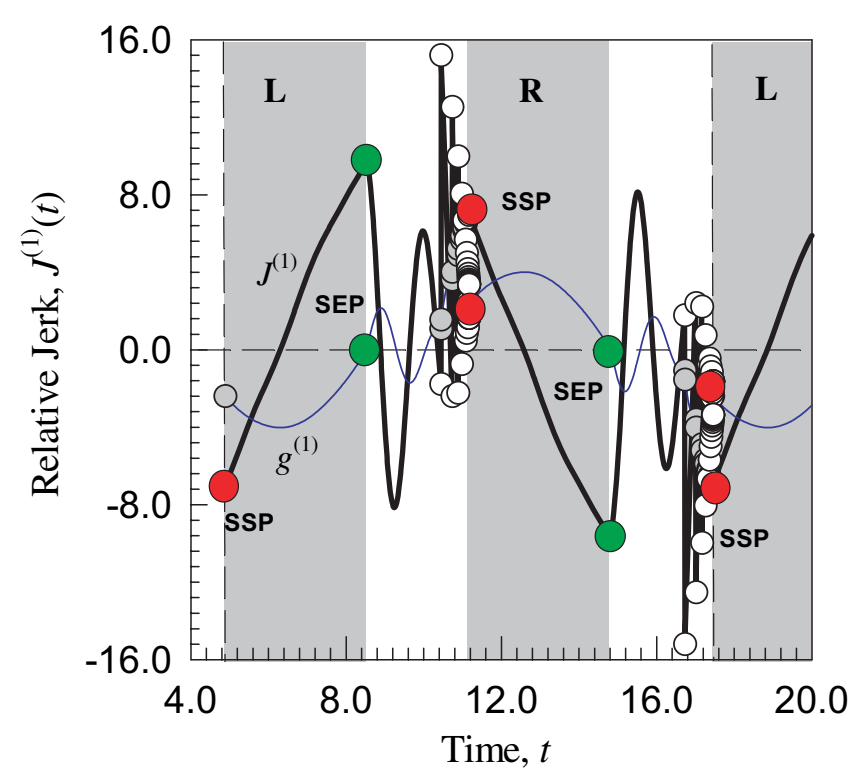

(d)

Fig. 11. Forces for the periodic motion of impacting chatter with stick $P_{645^{25}} 312^{25}$ : (a) relative force time-history and (b) internal forces for stick, (c) distributions along the displacement and (d) the relative jerk of the first gear $\left(m_{1}=2, m_{2}=1, r_{1}=\right.$ $r_{2}=0.6, k_{1}=30, k_{2}=20, Q_{0}=30, d=1.0, e=0.7$ and $\left.\Omega=0.5\right)$. The initial condition is $t_{0} \approx 4.8888960, x_{0}^{(1)} \approx-0.7387947$, $x_{0}^{(2)} \approx-0.2387947$ and $y_{0}^{(1)}=y_{0}^{(2)} \approx-0.2754161$.

$g_{\alpha}^{(i)}\left(t_{k}\right) \neq g_{\beta}^{(i)}\left(t_{k}\right), \alpha, \beta \in\{1,2,3\}$ and $\left.\alpha \neq \beta\right)$. From Luo and O'Connor [2007], the onset of the stick motion requires the two forces $g_{\alpha}^{(i)}\left(t_{k}\right)$ and $g_{\beta}^{(i)}\left(t_{k}\right)$ to have the same sign. To obtain the stick motion in $\Omega_{1}$ requires $g_{1}^{(1)}\left(t_{k}\right)<0$ and $g_{2}^{(1)}\left(t_{k}\right)<0$; and to obtain the stick motion in $\Omega_{3}$ requires $g_{1}^{(1)}\left(t_{k}\right)>0$ and $g_{2}^{(1)}\left(t_{k}\right)>0$. Such a relative force characteristic is presented in Fig. 11(a). In Fig. 11(b), the internal forces in the stick domains for two gears are presented because the internal forces between the two gears only exist for the stick. When the stick motion disappears, the internal forces vanish. In other words, the internal forces are zero at the 
vanishing of the stick motion. The vanishing condition for stick is $g_{\alpha}^{(i)}\left(t_{k}\right)=0$, which is observed in Figs. 11(a) and 11(b). To correspond to the trajectory in phase plane, the relative force distribution along the displacement of the first gear is shown in Fig. 11(c). The aforementioned conditions for the onset and vanishing of the stick motion are observed. However, this condition of $g_{\alpha}^{(i)}\left(\mathbf{x}_{\alpha}^{(i)}, t\right)=0$ is a necessary condition. The sufficient condition for the vanishing of the stick motion requires $J_{\alpha}^{(1)}\left(t_{k}\right)<0$ (or $J_{\alpha}^{(2)}\left(t_{k}\right)>0$ ) on the right and $J_{\alpha}^{(1)}\left(t_{k}\right)>0$ (or $J_{\alpha}^{(2)}\left(t_{k}\right)<0$ ) on the left side. Therefore, in Fig. 11(d), the jerk $J_{\alpha}^{(1)}(t)$ of the first gear is presented, and such a condition is observed. For instance, when the stick vanishes at $z_{k}^{(1)}=x_{k}^{(1)}-x_{k}^{(2)}=d / 2$, the relative velocity and acceleration are zero (i.e. $z_{k}^{(1)}=0$ and $\ddot{z}_{k}^{(1)}=0$ ). If $J_{\alpha}^{(1)}(t)<0$ for $t>t_{k}+\varepsilon$, the corresponding relative velocity and acceleration are greater than zero, which leads to the relative displacement satisfying $z^{(1)}=x^{(1)}-x^{(2)}<d / 2$. In other words, the two gears lie in the state of free-flying motion, and the stick disappears. The stick motion disappearance on the left side can be discussed in a similar manner. The jerk condition of $J_{\alpha}^{(1)}\left(t_{k}\right)>0$ leads to $z^{(1)}=x^{(1)}-x^{(2)}>-d / 2$. Therefore, the two gears start to be separated from the left side of the second gear, and the stick motion disappears.

\section{Conclusions}

In this paper, the analytical prediction of periodic motions relative to the impacting chatter with and without stick in the gear transmission system is completed through the mapping structure. The corresponding local stability and bifurcation analysis are carried out, and the grazing and stick conditions are used for the existence of periodic motions. Numerical simulations are performed to illustrate the periodic motions and stick motion criteria. The prediction of chaotic motions in such a gear system will be discussed in another paper.

\section{References}

Comparin, R. J. \& Singh, R. [1989] "Nonlinear frequency response characteristics of an impact pair," J. Sound Vibr. 134, 259-290.

den Hartog, J. P. \& Mikina, S. J. [1932] "Forced vibrations with non-linear spring constants," ASME J. Appl. Mech. 58, 157-164. di Bernardo, M., Budd, C. J. \& Champneys, A. R. [2001] "Normal form maps for grazing bifurcation in n-dimensional piecewise-smooth dynamical systems," Physica D 160, 222-254.

di Bernardo, M., Kowalczyk, P. \& Nordmark, A. B. [2002] "Bifurcation of dynamical systems with sliding: Derivation of normal form mappings, Physica $D$ 170, 175-205.

Han, R. P. S., Luo, A. C. J. \& Deng, W. [1995] "Chaotic motion of a horizontal impact pair," J. Sound Vibr. 181, 231-250.

Kahraman, A. \& Singh, R. [1990] "Nonlinear dynamics of a spur gear pair," J. Sound Vibr. 142, 49-75.

Karagiannis, K. \& Pfeiffer, F. [1991] "Theoretical and experimental investigations of gear box," Nonlin. Dyn. 2, 367-387.

Kleczka, M., Kreuzer, E. \& Schiehlen, W. [1992] "Local and global stability of a piecewise linear oscillator," Philos. Trans.: Phys. Sci. Engin. Nonl. Dyn. Engin. Syst. 338, 533-546.

Li, G. X., Rand, R. H. \& Moon, F. C. [1990] "Bifurcation and Chaos in a forced zero-stiffness impact oscillator," Int. J. Nonlin. Mech. 25, 417-432.

Luo, A. C. J. [2002] "An unsymmetrical motion in a horizontal impact oscillator," ASME J. Vibr. Acoust. 124, 420-426.

Luo, A. C. J. \& Menon, S. [2004] "Global chaos in a periodically forced, linear system with a deadzone restoring force," Chaos Solit. Fract. 19, 11891199.

Luo, A. C. J. [2005] "The mapping dynamics of periodic motions for a three-piecewise linear system under a periodic excitation," J. Sound Vibr. 283, 723-748.

Luo, A. C. J. \& Chen, L. D. [2005] "Periodic motion and grazing in a harmonically forced, piecewise, linear oscillator with impacts," Chaos Solit. Fract. 24, $567-578$.

Luo, A. C. J. [2006] Singularity and Dynamics on Discontinuous Vector Fields (Elsevier, Amsterdam).

Luo, A. C. J. \& Chen, L. D. [2007] "Arbitrary periodic motions and grazing switching of a forced piecewiselinear, impacting oscillator," ASME J. Vibr. Acoust. 129, 276-284.

Luo, A. C. J. \& O'Connor, D. [2007] "Nonlinear dynamics of a gear transmission dynamical system, Part I: Mechanism of impacting chatter and stick," Proc. IDETC'0\%, 2007 ASME International Design Engineering Conferences, September 4-7, 2007, Las Vegas, Nevada, USA. DETC2007-34881.

Menon, S. \& Luo, A. C. J. [2005] "A global period1 motion of a periodically forced, piecewise linear system," Int. J. Bifurcation and Chaos 15, 19451957.

Natsiavas, S. [1989] "Periodic response and stability of oscillators with symmetric trilinear restoring force," J. Sound Vibr. 134, 315-331. 
Nordmark, A. B. [1991] "Non-periodic motion caused by grazing incidence in an impact oscillator," J. Sound Vibr. 145, 279-297.

Pfeiffer, F. [1984] "Mechanische Systems mit unstetigen Ubergangen," Ing. Archiv 54, 232-240.

Rook, T. E. \& Singh, R. [1995] "Dynamic analysis of a reverse-idler gear pair with concurrent clearances," J. Sound Vibr. 182, 303-322.

Shaw, S. W. \& Holmes, P. J. [1983] "A periodically forced piecewise linear oscillator," J. Sound Vibr. 90, $129-155$.
Theodossiades, S. \& Natsiavas, S. [2000] "Non-linear dynamics of gear-pair systems with periodic stiffness and backlash," J. Sound Vibr. 229, 287-310.

\section{Appendix}

With an initial condition $\left(t, x^{(i)}, \dot{x}^{(i)}\right)=\left(t_{k}, x_{k}^{(i)}\right.$, $\left.y_{k}^{(i)}\right)$, the general solutions for two gear oscillators for three regions are given as follows:

Case I. $\left(d_{\alpha}^{(i)}\right)^{2}-c_{\alpha}^{(i)}>0$

$$
\begin{aligned}
x_{\alpha}^{(i)}= & e^{-d_{\alpha}^{(i)}\left(t-t_{k}\right)}\left(C_{1}^{(\alpha, i)} e^{\omega_{\alpha}^{(i)}\left(t-t_{k}\right)}+C_{2}^{(\alpha, i)} e^{-\omega_{d}^{(\alpha, i)}\left(t-t_{k}\right)}\right)+D_{1}^{(\alpha, i)} \cos \Omega t+D_{2}^{(\alpha, i)} \sin \Omega t+D_{0}^{(\alpha, i)}, \\
\dot{x}_{\alpha}^{(i)}= & e^{-d_{\alpha}^{(i)}\left(t-t_{k}\right)}\left[\left(\omega_{d}^{(\alpha, i)}-d_{\alpha}^{(i)}\right) C_{1}^{(\alpha, i)} e^{\omega_{d}^{(\alpha, i)}\left(t-t_{k}\right)}-\left(\omega_{d}^{(\alpha, i)}+d_{\alpha}^{(i)}\right) C_{2}^{(\alpha, i)} e^{-\omega_{d}^{(\alpha, i)}\left(t-t_{k}\right)}\right] \\
& -D_{1}^{(\alpha, i)} \Omega \sin \Omega t+D_{2}^{(\alpha, i)} \Omega \cos \Omega t ;
\end{aligned}
$$

where

$$
\left.\begin{array}{rl}
C_{1}^{(\alpha, i)}= & \frac{1}{2 \omega_{d}^{(\alpha, j)}}\left\{\dot{x}_{k}+\left(x_{k}-D_{0}^{(\alpha, i)}\right)\left(d_{\alpha}^{(i)}+\omega_{d}^{(\alpha, i)}\right)-\left[D_{1}^{(\alpha, i)}\left(d_{\alpha}^{(i)}+\omega_{d}^{(\alpha, i)}\right)+D_{1}^{(\alpha, i)} \Omega\right] \cos \Omega t_{k}\right. \\
& \left.+\left[D_{1}^{(\alpha, i)} \Omega-D_{2}^{(\alpha, i)}\left(d_{\alpha}^{(i)}+\omega_{d}^{(\alpha, i)}\right)\right] \sin \Omega t_{k}\right\}, \\
C_{1}^{(\alpha, i)}= & \frac{1}{2 \omega_{d}^{(\alpha, j)}}\left\{-\dot{x}_{k}+\left(x_{k}-D_{0}^{(\alpha, i)}\right)\left(-d_{\alpha}^{(i)}+\omega_{d}^{(\alpha, i)}\right)-\left[D_{1}^{(\alpha, i)} \Omega+D_{2}^{(j)}\left(-d_{\alpha}^{(i)}+\omega_{d}^{(\alpha, i)}\right)\right] \sin \Omega t_{k}\right\} \\
& \left.-\left[D_{1}^{(\alpha, i)}\left(-d_{\alpha}^{(i)}+\omega_{d}^{(\alpha, i)}\right)-D_{2}^{(\alpha, i)} \Omega\right] \cos \Omega t_{k}\right\}, \\
D_{0}^{(\alpha, i)}= & -\frac{b_{\alpha}^{(i)}}{c_{\alpha}^{(i)}}, \quad D_{1}^{(\alpha, i)}=\frac{Q_{0}^{(i)}\left(c_{\alpha}^{(i)}-\Omega^{2}\right)}{\left(c_{\alpha}^{(i)}-\Omega^{2}\right)^{2}+\left(2 d_{\alpha}^{(i)} \Omega\right)^{2}}, \\
D_{2}^{(\alpha, i)}= & \frac{Q_{0}^{(i)}\left(2 d_{\alpha}^{(i)} \Omega\right)}{\left(c_{\alpha}^{(i)}-\Omega^{2}\right)^{2}+\left(2 d_{\alpha}^{(i)} \Omega\right)^{2}}, \quad \omega_{d}^{(\alpha, i)}=\sqrt{\left(d_{\alpha}^{(i)}\right)^{2}-c_{\alpha}^{(i)}} .
\end{array}\right\}
$$

Case II. $\left(d_{\alpha}^{(i)}\right)^{2}-c_{\alpha}^{(i)}<0$

$$
\left.\begin{array}{rl}
x^{(i)}= & e^{-d_{\alpha}^{(i)}\left(t-t_{i}\right)}\left[C_{1}^{(\alpha, i)} \cos \omega_{d}^{(j)}\left(t-t_{k}\right)+C_{2}^{(\alpha, i)} \sin \omega_{d}^{(j)}\left(t-t_{k}\right)\right]+D_{1}^{(\alpha, i)} \cos \Omega t+D_{2}^{(\alpha, i)} \sin \Omega t+D_{0}^{(\alpha, i)}, \\
\dot{x}^{(i)}= & e^{-d_{\alpha}^{(i)}\left(t-t_{i}\right)}\left[\left(C_{2}^{(\alpha, i)} \omega_{d}^{(\alpha, i)}-d_{\alpha}^{(i)} C_{1}^{(\alpha, i)}\right) \cos \omega_{d}^{(\alpha, i)}\left(t-t_{k}\right)-\left(C_{1}^{(\alpha, i)} \omega_{d}^{(\alpha, i)}+d_{\alpha}^{(i)} C_{2}^{(\alpha, i)}\right) \sin \omega_{d}^{(\alpha, i)}\left(t-t_{k}\right)\right] \\
& -D_{1}^{(\alpha, i)} \Omega \sin \Omega t+D_{2}^{(\alpha, i)} \Omega \cos \Omega t
\end{array}\right\}
$$

where

$$
\left.\begin{array}{l}
C_{1}^{(\alpha, i)}=x_{k}-D_{1}^{(\alpha, i)} \cos \Omega t_{k}-D_{2}^{(\alpha, i)} \sin \Omega t_{k}-D_{0}^{(\alpha, i)}, \\
\left.C_{2}^{(\alpha, i)}=\frac{1}{\omega_{d}^{(j)}}\left[d_{\alpha}^{(i)}\left(x_{k}-D_{1}^{(\alpha, i)} \cos \Omega t_{k}-D_{2}^{(\alpha, i)} \sin \Omega t_{k}-D_{0}^{(\alpha, i)}\right)+\dot{x}_{k}+D_{1}^{(\alpha, i)} \Omega \sin \Omega t_{k}-D_{2}^{(\alpha, i)} \Omega \cos \Omega t_{k}\right)\right], \\
\omega_{d}^{(\alpha, i)}=\sqrt{c_{\alpha}^{(i)}-\left(d_{\alpha}^{(i)}\right)^{2}} .
\end{array}\right\}
$$


Case III. $\left(d_{\alpha}^{(i)}\right)^{2}-c_{\alpha}^{(i)}=0$

$$
\left.\begin{array}{l}
x^{(i)}=e^{-d_{\alpha}^{(i)}\left(t-t_{i}\right)}\left[C_{1}^{(\alpha, i)}\left(t-t_{i}\right)+C_{2}^{(\alpha, i)}\right]+D_{1}^{(\alpha, i)} \cos \Omega t+D_{2}^{(\alpha, i)} \sin \Omega t+D_{0}^{(\alpha, i)}, \\
\dot{x}^{(i)}=e^{-d_{\alpha}^{(i)}\left(t-t_{i}\right)}\left[C_{1}^{(\alpha, i)}-C_{1}^{(\alpha, i)} d_{\alpha}^{(i)}\left(t-t_{i}\right)-d_{\alpha}^{(i)} C_{2}^{(\alpha, i)}\right]-D_{1}^{(\alpha, i)} \Omega \sin \Omega t+D_{2}^{(\alpha, i)} \Omega \cos \Omega t ;
\end{array}\right\}
$$

where

$$
\left.\begin{array}{l}
C_{2}^{(\alpha, i)}=x_{k}-D_{1}^{(\alpha, i)} \cos \Omega t_{k}-D_{2}^{(\alpha, i)} \sin \Omega t_{k}-D_{0}^{(\alpha, i)} \\
C_{2}^{(\alpha, i)}=x_{k}+\left(D_{2}^{(\alpha, i)} \Omega-d_{\alpha}^{(i)} D_{1}^{(\alpha, i)}\right) \cos \Omega t_{k}-\left(D_{1}^{(\alpha, i)} \Omega+d_{\alpha}^{(i)} D_{2}^{(\alpha, i)}\right) \sin \Omega t_{k}-d_{\alpha}^{(i)} D_{1}^{(\alpha, i)} .
\end{array}\right\}
$$

Case $\boldsymbol{I} \boldsymbol{V} . d_{\alpha}^{(i)} \neq 0, c_{\alpha}^{(i)}=0$

$$
\left.\begin{array}{l}
x^{(i)}=C_{1}^{(\alpha, i)} e^{-2 d_{\alpha}^{(i)}\left(t-t_{k}\right)}+D_{1}^{(\alpha, i)} \cos \Omega t+D_{2}^{(\alpha, i)} \sin \Omega t+D_{0}^{(\alpha, i)} t+C_{2}^{(\alpha, i)}, \\
\dot{x}^{(i)}=-2 d_{\alpha}^{(i)} C_{1}^{(\alpha, i)} e^{-2 d_{\alpha}^{(i)}\left(t-t_{k}\right)}-D_{1}^{(\alpha, i)} \Omega \sin \Omega t+D_{2}^{(\alpha, i)} \Omega \cos \Omega t+D_{0}^{(\alpha, i)} ;
\end{array}\right\}
$$

where

$$
\left.\begin{array}{rl}
C_{1}^{(\alpha, i)}= & -\frac{1}{2 d_{\alpha}^{(i)}}\left(\dot{x}_{k}+D_{1}^{(\alpha, i)} \Omega \sin \Omega t_{k}-D_{2}^{(\alpha, i)} \Omega \cos \Omega t_{k}-D_{0}^{(\alpha, i)}\right), \\
C_{2}^{(\alpha, i)}= & \frac{1}{2 d_{\alpha}^{(i)}}\left[2 d_{\alpha}^{(i)} x_{k}+\dot{x}_{k}+\left(D_{1}^{(\alpha, i)} \Omega-2 d_{\alpha}^{(i)} D_{2}^{(\alpha, i)}\right) \sin \Omega t_{k}-\left(2 d_{\alpha}^{(i)} D_{1}^{(\alpha, i)}+D_{2}^{(\alpha, i)} \Omega\right) \cos \Omega t_{k}\right. \\
& \left.-2 d_{\alpha}^{(i)} D_{0}^{(\alpha, i)} t_{k}-D_{0}^{(\alpha, i)}\right] .
\end{array}\right\}
$$

Case $V \cdot d_{\alpha}^{(i)}=0, c_{\alpha}^{(i)}=0$

$$
\left.\begin{array}{l}
x^{(i)}=-\frac{Q_{0}^{(i)}}{\Omega^{2}} \cos \Omega t-\frac{1}{2} b_{\alpha}^{(i)} t^{2}+C_{1}^{(\alpha, i)} t+C_{2}^{(\alpha, i)}, \\
\dot{x}^{(i)}=\frac{Q_{0}^{(i)}}{\Omega} \sin \Omega t-b_{\alpha}^{(i)} t+C_{1}^{(\alpha, i)} ;
\end{array}\right\}
$$

where

$$
\left.\begin{array}{l}
C_{1}^{(\alpha, i)}=\dot{x}_{k}-\frac{Q_{0}^{(i)}}{\Omega} \sin \Omega t_{k}+b_{\alpha}^{(i)} t_{k}, \\
C_{2}^{(\alpha, i)}=x_{k}-\dot{x} t_{k}+\frac{Q_{0}^{(i)}}{\Omega^{2}} \cos \Omega t_{k}+\frac{Q_{0}^{(i)}}{\Omega} t_{k} \sin \Omega t_{k}-\frac{1}{2} b_{\alpha}^{(i)} t_{k}^{2} \cdot
\end{array}\right\}
$$

